\title{
Concentric Eyewall Formation in Typhoon Sinlaku (2008). Part III: Horizontal Momentum Budget Analyses
}

\author{
Yi-HSUAN HuANG AND CHUN-CHIEH WU \\ Department of Atmospheric Sciences, National Taiwan University, Taipei, Taiwan \\ MiCHAEL T. MONTGOMERY \\ Department of Meteorology, Naval Postgraduate School, Monterey, California
}

(Manuscript received 30 January 2018, in final form 3 July 2018)

\begin{abstract}
This is a follow-up work to two prior studies examining secondary eyewall formation (SEF) in Typhoon Sinlaku (2008). This study shows that, in the SEF region, the majority of the elevated winds are supergradient. About two-thirds of the rapid increase in tangential wind tendencies immediately prior to SEF are attributed to agradient wind tendencies. This suggests the importance of nonlinear, unbalanced dynamical processes in $\mathrm{SEF}$ in addition to the classical axisymmetric balanced response to forcings of heating and momentum. In the SEF region, analyses show two distinct responsible processes for the increasing azimuthal tangential wind in two vertical intervals. Within the boundary inflow layer, the competing effect between the mean radial influx of absolute vorticity and deceleration caused by surface friction and subgrid diffusion yields a secondary maximum of positive tendency. Analyses further demonstrate the major impact of the mean radial influx of absolute vorticity on SEF. Above the boundary inflow layer, the vertical advection acts to vertically extend the tangential wind jet via the lofting of the enhanced tangential momentum farther upward. The roles of the nonlinear unbalanced dynamics in these two processes are discussed in this paper. From a Lagrangian perspective, the persistently increasing agradient force outweighs the frictional loss, effectively decelerating boundary layer inflowing air across the SEF region. This explains the sharpening of the radial gradient of boundary layer inflow, which is shown to be responsible for the buildup of a zone with concentrated boundary layer convergence. The previously proposed unbalanced dynamical pathway to SEF is elaborated upon and supported by the current results and discussion.
\end{abstract}

\section{Introduction}

The double eyewall structure in tropical cyclones (TCs) is a phenomenon that features two concentric quasi-circular deep convective rings, representing the two eyewalls, and a nearly cloud-free region between the eyewalls (the moat). Such concentric eyewall structures have been found to be common in intense TCs and have been occasionally documented in weaker TCs from satellite imagery and aircraft observations (Hawkins and Helveston 2008; Kossin and Sitkowski 2009; Kuo et al. 2009; Yang et al. 2013). The widely documented association between the occurrence of concentric eyewalls and short-term changes in TC intensity and structure (Willoughby et al. 1982; Black and

\footnotetext{
Corresponding author: Chun-Chieh Wu, cwu@typhoon.as.ntu. edu.tw
}

Willoughby 1992; Willoughby and Black 1996; Houze et al. 2006, 2007; Hawkins and Helveston 2008; Kossin and Sitkowski 2009; Kuo et al. 2009; Didlake and Houze 2011; Sitkowski et al. 2011, 2012; Bell et al. 2012; Hence and Houze 2012; Yang et al. 2013; Abarca et al. 2016; Didlake et al. 2017) makes the secondary eyewall formation (SEF; referred to as the outer-eyewall formation) and its subsequent eyewall replacement cycle an important problem in tropical severe weather forecasting and atmospheric science.

Various dynamical and thermodynamical pathways to SEF have been proposed in the literature. However, the relative importance of each proposed mechanism still requires further evaluation. The first paper of this series of studies (Wu et al. 2012) and a recent book chapter (Wu et al. 2015) extensively reviewed the possible mechanisms for SEF. Regarding dynamical processes intrinsic to the vortex, scientific discussions have been 
stimulated by and focused on the importance of axisymmetric versus nonaxisymmetric (eddy) dynamics and of balanced versus unbalanced dynamics (particularly, the disruption of gradient wind balance in the frictional boundary layer of a tropical cyclone vortex).

From an axisymmetric perspective, the role of unbalanced flow within the boundary layer and just above the boundary layer top was first addressed by two companion studies, Wu et al. (2012) and Huang et al. (2012, hereafter HMW12). Together, the findings of these studies point to collective structural changes in the outer-core region in support of the continued enhancement of ascent out of the boundary layer, culminating ultimately in the formation of a secondary eyewall. Around 1 day prior to SEF, the broadening of the lowlevel tangential wind field in the storm's outer-core region is found to be associated with the intensification of the eyewall and the persistently strengthening boundary layer inflow. The broadening swirling flow can be approximately explained by the balanced response to the radial gradient of diabatic heating (Schubert and Hack 1982; Shapiro and Willoughby 1982; Hack and Schubert 1986; Bui et al. 2009). In the region where SEF subsequently forms (the SEF region), HMW12 then identified the close connection between the organization of convection and the progressive increase of a positive agradient force in the radial direction in response to the broadening tangential winds and the intensifying boundary layer inflow. In a convectively favorable environment, a progressive increase in the positive agradient force provides a persistent mechanical means for air to erupt from the boundary layer in the SEF region. HMW12 argued that the development of such a region would generally lead to the focusing and sustenance of deep convection in the narrow supergradient wind zone. This proposed pathway to SEF is referred to as the unbalanced dynamical pathway to SEF in this article.

A number of studies have examined the unbalanced dynamical pathway to SEF in HMW12 from different perspectives. The development of a narrow supergradient wind zone in the SEF region has been identified in numerical simulations for real TCs or for a vortex with idealized design and a quiescent environment (Abarca and Montgomery 2013; Qiu and Tan 2013; Wang et al. 2013; Sun et al. 2013; Wang et al. 2016). Given that rainbands are prevalent in the vortex's outer-core region prior to SEF, Qiu and Tan (2013) suggested the impact of the associated asymmetric agradient forces on the downstream boundary layer flow and subsequently on SEF. While evaluating the depth-integrated boundary layer flow, Wang et al. (2013) demonstrated that the supergradient and frictional forces are the two leading terms contributing to the secondary maximum in the boundary layer convergence, providing supporting evidence for the unbalanced dynamical pathway to SEF.

The unbalanced flow in the secondary eyewall has been documented in aircraft observations as well. With the data collected during the Hurricane Rainband and Intensity Change Experiment (RAINEX; Houze et al. 2006), the local maxima of supergradient flow were found in Rita's primary and secondary eyewalls (Didlake and Houze 2011). Bell et al. (2012) further showed that the location where the boundary layer inflow decelerates coincides with the supergradient wind region. More recently, the secondary supergradient wind zone that coincides with the SEF region was first documented during the SEF period in an observationally based study of Hurricane Edouard (2014) by Abarca et al. (2016). Edouard was intensively observed during the National Aeronautics and Space Administration (NASA) Hurricane and Severe Storm Sentinel (HS3; Braun et al. 2016) experiment.

Beginning with a broad tangential wind profile as an initial condition, Abarca and Montgomery (2013, their section 6) used a simple time-dependent slab boundary layer model to illustrate the tendency of the frictional boundary layer dynamics to progressively control the initiation of a secondary eyewall. To illustrate the basic idea, they used a fixed radial pressure gradient inferred from the full-physics model at a time just before the emergence of a secondary tangential wind maximum. Of course, fixing the radial pressure gradient is not realistic, but the results nonetheless served to highlight the intrinsic capability of the nonlinear boundary layer dynamics to initiate a secondary maximum of tangential velocity, upward motion, and boundary layer convergence in the boundary layer under the idealized assumption of an active eyewall that maintains the swirling wind field (and its associated radial pressure gradient) above the boundary layer.

Deactivating all the model physics, except the planetary boundary layer process, Menelaou et al. (2014) ${ }^{1}$ carried out a Weather Research and Forecasting (WRF) Model simulation in which the vortex continuously weakens and does not undergo SEF, arguing that the boundary layer dynamics is not essential for SEF. Nevertheless, results ruling out sole physical processes deviate from the scientific question addressed in HMW12 and other related studies, which attempted to address

\footnotetext{
${ }^{1}$ As already noted by Smith and Montgomery (2015, p. 3027), "the recent study by Menelaou et al. (2014) appears to have challenged the application of the new intensification paradigm to the problem of secondary eyewall formation and the role of the frictional boundary layer dynamics... . In their sensitivity experiment with heating turned off, they have simply rediscovered that a vortex without sustained forcing [emphasis added] will spin down."
} 
the competing effects among each physical processes. In addition, given that the concentric eyewall structure is widely acknowledged as a phenomenon identified mainly in mature TCs near its peak intensity (Sitkowski et al. 2011; Yang et al. 2013; Zhou and Wang 2013), it is not surprising that SEF does not occur in weak and continuously weakening storms.

The role of axisymmetric balanced dynamics in SEF has been qualitatively and quantitatively studied using the Sawyer-Eliassen balance model (Eliassen 1951; Bui et al. 2009, and references) or an alternative method using a linearized primitive-equation formulation and a long-time limit ${ }^{2}$ [e.g., the Three-Dimensional Vortex Perturbation Analysis and Simulation (3DVPAS) described in Hodyss and Nolan (2007) and Nolan et al. (2007)]. These diagnostic models offer a classical perspective on how the slow transverse circulation responds to a given diabatic heating and tangential momentum forcing. In balance dynamics, the slow transverse circulation acts to restore thermal wind balance. Tangential wind tendencies caused by the derived transverse circulation are evaluated accordingly. In Rozoff et al. (2012), the diagnosed results were used to argue that the expansion of kinetic energy (or the enhanced inertial stability), together with sustained diabatic heating, are important to the enhancement of tangential winds in the SEF region. The enhanced inertial stability is consistent with the beta-skirt structure (Terwey and Montgomery 2008), vorticity skirt (Kuo et al. 2008), and the expansion of tangential winds (Wu et al. 2012; HMW12; Bell et al. 2012). In addition to Rozoff et al. (2012), the Sawyer-Eliassen integrations in Sun et al. (2013) and Wang et al. (2016) presented results supporting that balanced dynamics can explain the increment in the tangential wind tendency above the boundary layer in the SEF region prior to SEF. Sun et al. (2013) demonstrated the dominant impact of the outer rainband heating, as compared with the eyewall heating, on enhancing tangential winds in the SEF region. Zhu and Zhu (2014) further demonstrated that the low-level heating (at the lowest $3 \mathrm{~km}$ ) has a profound effect on the enhancement of tangential winds and the development of boundary layer convergence in the SEF region upon SEF.

Taking a different point of view from HMW12, Kepert (2013) suggested that the initial updraft complex in the secondary eyewall can be elementally understood from a quasi-linear Ekman pumping law based on the assumption that the flow in the boundary layer is not far from

\footnotetext{
${ }^{2}$ Recent work has noted the limitation of both approaches on account of the neglect of nonlinear terms in the inner-core boundary layer and eyewall of the vortex (Montgomery and Smith 2017; Abarca and Montgomery 2015).
}

a state of gradient wind balance in a first approximation. Applying a family of diagnostic boundary layer models, Kepert (2013) suggested that both the Ekman updraft in response to a small radial gradient of vorticity at the outer radii of a vortex and the hypothesized feedback between this frictionally forced updraft and the subsequent convectively generated vorticity contribute to SEF. Subsequently, Montgomery et al. (2014) and Kepert and Nolan (2014) examined the mechanism proposed by Kepert (2013) using the diagnosed TC structure from numerical simulations with sophisticated model physics. Montgomery et al. (2014) articulated several concerns with the SEF feedback mechanism using the zeroth-order Ekman pumping formulation for the updraft complex and presented quantitative calculations to support those concerns. Montgomery et al. (2014) found that the (unfiltered) quasi-linear Ekman solution produced unrealistic multiple pairs of updrafts and downdrafts in and near the SEF region. Kepert and Nolan (2014) purportedly countered the critique of Montgomery et al. (2014) by emphasizing the nonlinear aspects of the boundary layer dynamics [many of which were emphasized also by Montgomery et al. (2014)]. Kepert and Nolan (2014) and Kepert (2017) showed that the applied nonlinear boundary layer model is insensitive to small-scale perturbations in the applied TC structure and concluded that these small perturbations should not refute the hypothesized feedback mechanism of Kepert (2013). Kepert (2017) later demonstrated that the frictionally induced updraft tends to bring the presupposed vorticity bump at the top of the boundary layer at larger radii radially inwards and to locally amplify the vorticity at smaller radii. Looking anew at the foregoing studies, both studies would seem to share the view of an intrinsic process of SEF involving a progressive boundary layer control on the emerging outer eyewall.

Keeping an open mind about the relative importance of the balanced, quasi-balanced, and nonlinear unbalanced dynamics in SEF, this study presents new tests of the dynamical SEF pathway proposed by HMW12. This study is specifically aimed to identify the dynamical processes responsible for 1) the formation of the secondary azimuthalmean tangential wind maximum within and near the top of the boundary layer (Fig. 1a) and 2) the increase in the radial gradient of the azimuthal-mean boundary layer inflow and associated convergence in the boundary layer (Fig. 1b). Analyses of the averaged tangential wind tendency equation and averaged material derivative of the radial velocity are conducted to shed new insight and answers to the foregoing questions, respectively.

Although HMW12 and this study emphasize the dynamical processes within and just above the boundary layer, the presented results do not, by themselves, imply 
(a) tangential velocity
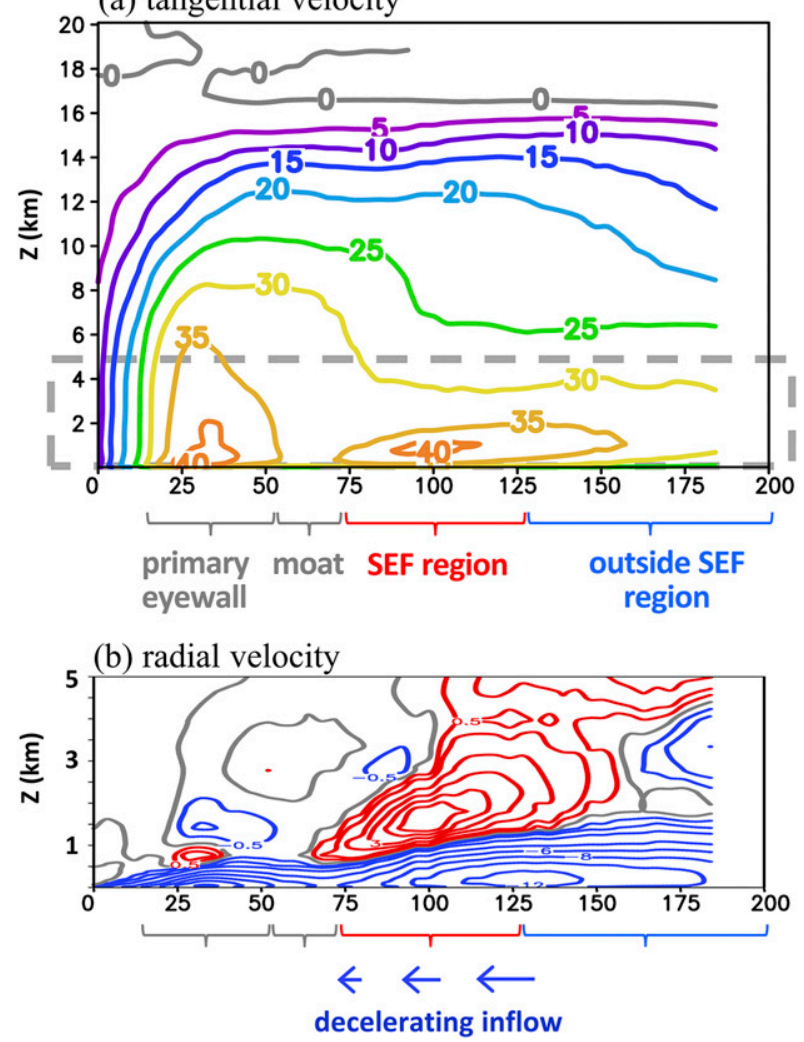

FIG. 1. Radius-height cross sections of the azimuthally averaged (a) tangential velocity from the ocean surface to the model top and (b) radial velocity (red: outflow; blue: inflow; gray: $0 \mathrm{~m} \mathrm{~s}^{-1}$ ) in the lowest $5 \mathrm{~km}$ [highlighted by the dashed box in (a)] at $2 \mathrm{~h}$ after SEF. Contour intervals of tangential and radial velocity are 5 and $2 \mathrm{~m} \mathrm{~s}^{-1}$, respectively. Additional contours of $\pm 0.5 \mathrm{~m} \mathrm{~s}^{-1}$ are plotted in (b).

that other factors, such as the diabatic heating associated with rainbands, are irrelevant. The role of rainband heating in SEF lies outside the scope of the current study and merits further investigation, as noted later in the conclusions.

The remaining manuscript is organized as follows. Section 2 briefly introduces the data used to carry out the horizontal momentum budget analyses. The specific equations used to diagnose the model simulations are presented in section 3. Section 4 highlights the intrinsic flow characteristics of interest, including the agradient component of the TC structure and the sharpening of the radial gradient of inflow (and convergence) in the boundary layer. Results of the momentum budget calculations along with their comparisons to previous research are presented in section 5. A discussion of the main findings is provided in section 6. Section 7 gives the conclusions.

\section{Data}

The data used in this study are the same as those presented in HMW12. Flight observations collected during The Observing System Research and Predictability Experiment (THORPEX) Pacific Asian Regional Campaign (T-PARC; Elsberry and Harr 2008) provides exceptionally valuable information on the storm intensity and structure of Typhoon Sinlaku (2008). Applying the EnKF data assimilation system (Meng and Zhang 2007, 2008a,b), three special observation operators innovated in $\mathrm{Wu}$ et al. (2010), and the WRF Model (Skamarock et al. 2005), Wu et al. (2012) constructed a dataset with satisfactory spatial resolution and high temporal resolution to represent the observed life cycle of Sinlaku. The dataset contains simulations integrated for more than 4 days, starting from 0300 UTC 9 September. The simulated results are in good agreement with the observations, containing depictions of the overall evolution of the TC movement and changes in intensity and structure, including Sinlaku's SEF. Different variables were examined from various viewpoints to determine the SEF time, which was identified to occur around 0700 UTC 11 September. In addition to the broadening of tangential winds, which was noted about 1 day prior to Sinlaku's SEF in Wu et al. (2012), in a follow-up paper, HMW12 discussed the importance of the concurrent flow evolution featuring the increasing boundary layer inflow in the vortex's outer-core region. In the SEF region $(75-125 \mathrm{~km})$, they noted the development of supergradient winds in the upper boundary layer and lower troposphere below about $2 \mathrm{~km}$, the concentration of boundary layer convergence, and the enhanced ascent near the top of the boundary layer (their Figs. 5 and 6). HMW12 provided dynamical links among these identified flow characteristics and SEF and presented a new pathway to SEF from the azimuthally averaged perspective.

To obtain a more complete understanding of the dynamical processes supporting the observed and simulated SEF event, this study conducts a detailed horizontal momentum budget diagnosis by rerunning the simulations to produce 2-min output data. Data from the innermost domain, at 5-km horizontal grid spacing, are used for the diagnostic analyses herein. In accord with the azimuthally averaged viewpoint of the SEF problem adopted in this study, all state variables are interpolated to a cylindrical coordinate system centered on the circulation center of the storm.

\section{Horizontal momentum equations}

\section{a. Tangential velocity tendency equation}

In a cylindrical coordinate centered on the vortex center, the azimuthally averaged tangential velocity equation is as follows: 


$$
\frac{\partial \bar{v}}{\partial t}=-\bar{u} \overline{\zeta_{a}}-\bar{w} \frac{\partial \bar{v}}{\partial z}-\overline{u^{\prime} \zeta_{a}^{\prime}}-\overline{w^{\prime} \frac{\partial v^{\prime}}{\partial z}}-\overline{\frac{1}{\rho r} \frac{\partial P^{\prime}}{\partial \lambda}}+\bar{F}_{\lambda},
$$

where $t$ denotes time; $u, v$, and $w$ refer to the radial, tangential, and vertical components of velocity, respectively; $\zeta_{a}$ denotes the vertical component of absolute vorticity, $\zeta_{a}=\zeta+f$, where $\zeta$ and $f$ are the vertical components of relative vorticity and the Coriolis parameter, respectively; $\zeta=(v / r+\partial v / \partial r-\partial u / r \partial \theta)$, where the first two terms on the right-hand side are curvature vorticity and shear vorticity, respectively. The overline symbol over a parameter represents its azimuthalmean quantity; the apostrophe represents the corresponding asymmetric component (eddy). The left-hand side of Eq. (1) is the local tendency of mean tangential velocity. The first and third terms on the right-hand side are the radial fluxes of absolute vorticity due to the azimuthal-mean flow and eddies. The second and fourth terms are the mean and eddy components of the vertical advection of tangential velocity. The fifth term is the azimuthal mean of the tangential component of the perturbation pressure gradient force. The final term $\bar{F}_{\lambda}$ stands for the effect due to surface friction and subgrid-scale diffusion, parameterized by the selected PBL and surface schemes; it is derived from the zonal and meridional components of the subgridscale flux divergence terms, which are explicitly output at 2-min intervals from the WRF Model during the integration.

\section{b. Material derivative of radial velocity}

In a cylindrical coordinate system centered on a suitable vortex circulation center, the azimuthally averaged material derivative of the radial velocity $\langle d u / d t\rangle$ is as follows:

$$
\left\langle\frac{d u}{d t}\right\rangle=-\frac{1}{\bar{\rho}} \frac{\partial \bar{P}}{\partial r}+f \bar{v}+\frac{\bar{v}^{2}}{r}-\frac{\overline{1} \frac{\partial P^{\prime}}{\rho}}{\partial r}+\frac{\overline{v^{\prime 2}}}{r}+\overline{F_{r}}
$$

where $P$ is the pressure and $\rho$ is the density. The last term $F_{r}$ is the effect of the PBL parameterization and surface scheme, derived from the zonal and meridional components of the subgrid-scale flux divergence terms, which are explicitly output at 2-min intervals from the WRF Model during the integration. An overbar denotes an azimuthal average. The first and fourth terms on the right-hand side are the azimuthal mean of the radial component of the pressure gradient force due to the axisymmetric and eddy structure, respectively. The second term presents the Coriolis force. The third and fifth terms are the azimuthalmean centrifugal force due to the mean flow and eddy, respectively. A positive (negative) value stands for a radially outward (inward) forcing, which acts to decelerate (accelerate) inflowing air parcels or accelerate (decelerate) outflowing air parcels. The azimuthal average of the agradient force $\langle\mathrm{AF}\rangle$ is the sum of the first five terms in Eq. (2). Equation (2) then becomes

$$
\left\langle\frac{d u}{d t}\right\rangle=\langle\mathrm{AF}\rangle+\overline{F_{r}}
$$

The contributions of the mean and eddy structure to $\langle\mathrm{AF}\rangle$ are denoted by mean agradient force $(\mathrm{mAF})$ and eddy agradient force (eAF), respectively, where

$$
\begin{aligned}
\mathrm{mAF} & =-\frac{1}{\bar{\rho}} \frac{\partial \bar{P}}{\partial r}+f \bar{v}+\frac{\bar{v}^{2}}{r} \quad \text { and } \\
\mathrm{eAF} & =-\frac{\overline{1}}{\rho} \frac{\partial P^{\prime}}{\partial r}+\frac{\overline{v^{\prime 2}}}{r} .
\end{aligned}
$$

\section{Overview of some key flow characteristics}

\section{a. Agradient flow component}

Figure 2 illustrates the height-radius cross sections of the mean agradient force and the azimuthal-mean radial flow before, during, and after the SEF. Results with a consistently evolving low-level structure of the mean agradient force and radial flow outside the eyewall are collected consecutively to compute the temporal average. Therefore, the time spans for Figs. $2 \mathrm{a}$ and $2 \mathrm{~b}$ are different from the others. Regions of positive mean agradient forces are referred to as "supergradient force" patches or zones.

About 1 day before SEF, isolated and weak supergradient force patches wax and wane outside the primary eyewall as outer rainbands form and dissipate, leaving a zone of weak supergradient forces between 100 and $125 \mathrm{~km}$ in the average between $\mathrm{H}-37$ and $\mathrm{H}-25$ (Fig. 2a). This weak supergradient force patch later dissipates, and meanwhile, an area of weak supergradient forces extends radially outward from the eyewall at H-25-H-19 (Fig. 2b). This area subsequently expands radially outward and evolves into two supergradient force patches outside the eyewall between $\mathrm{H}-19$ and H-16 (Fig. 2c). Then the two patches merge with each other at around $60-110 \mathrm{~km}$ and separate from the eyewall during H-16-H-13 (Fig. 2d). Subsequently, the supergradient force strengthens and concentrates in the SEF region persistently in time to the SEF event (Figs. 2e-i). This supergradient force zone steadily extends outwards toward $150 \mathrm{~km}$ after $\mathrm{H}-07$ (Figs. $2 \mathrm{~g}-\mathrm{i}$ ), while its magnitude beyond $125 \mathrm{~km}$ remains marginal $\left(<6 \mathrm{~m} \mathrm{~s}^{-1} \mathrm{~h}^{-1}\right)$. The radius of the secondary maximum azimuthal-mean tangential wind is close to the radii 
(a) $\mathrm{H}-37-\mathrm{H}-25$

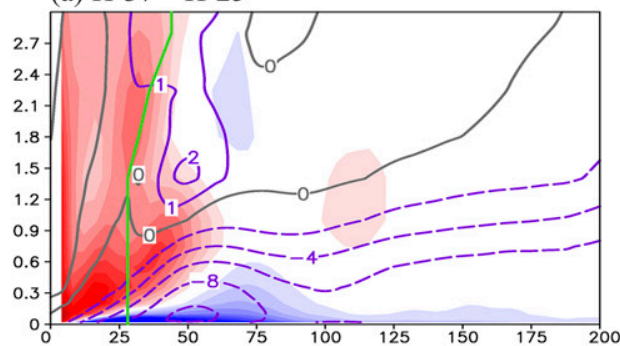

(c) $\mathrm{H}-19-\mathrm{H}-16$

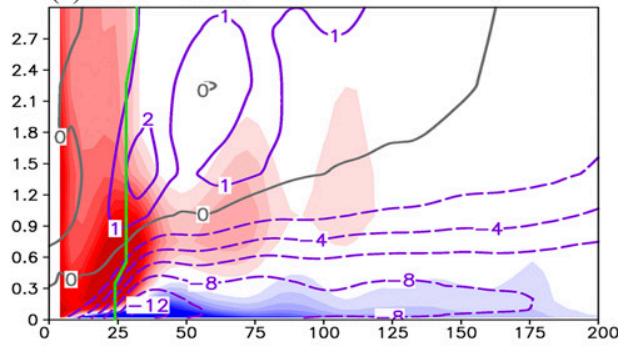

(e) $\mathrm{H}-13-\mathrm{H}-10$

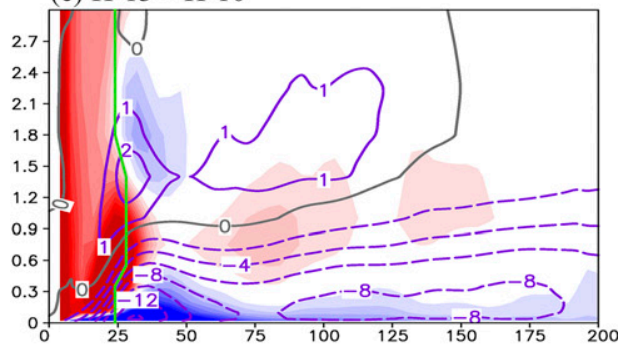

(g) $\mathrm{H}-07-\mathrm{H}-04$

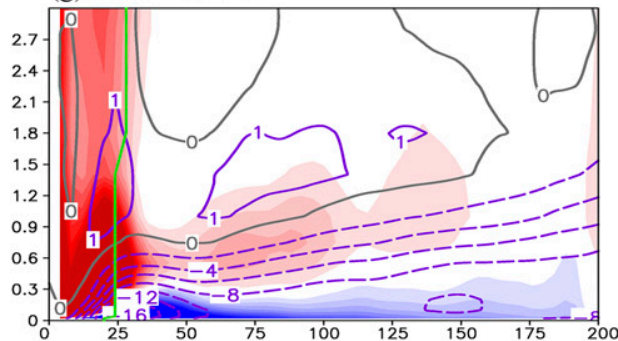

(i) $\mathrm{H}-01-\mathrm{SEF}$

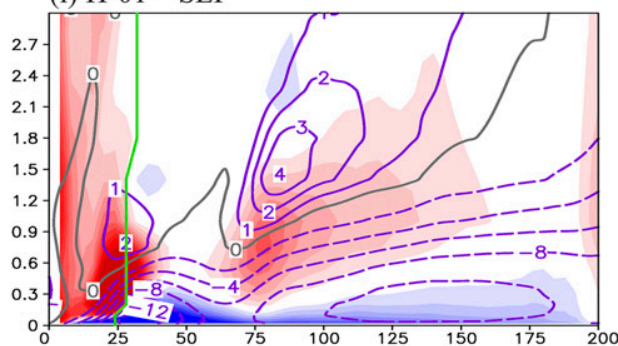

(b) $\mathrm{H}-25-\mathrm{H}-19$

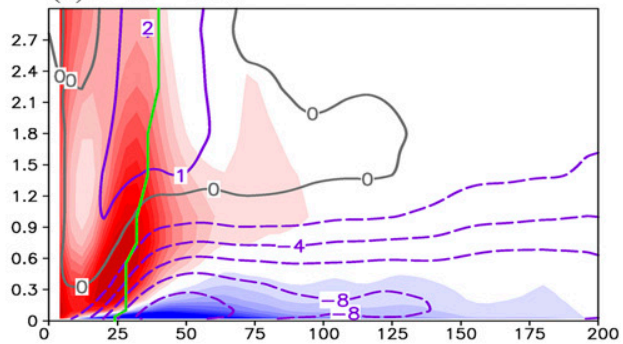

(d) $\mathrm{H}-16-\mathrm{H}-13$

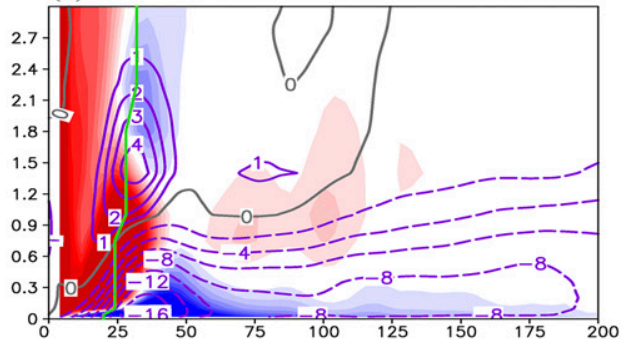

(f) $\mathrm{H}-10-\mathrm{H}-07$

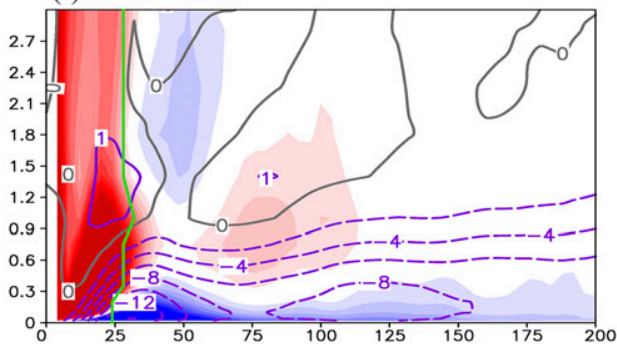

(h) $\mathrm{H}-04-\mathrm{H}-01$

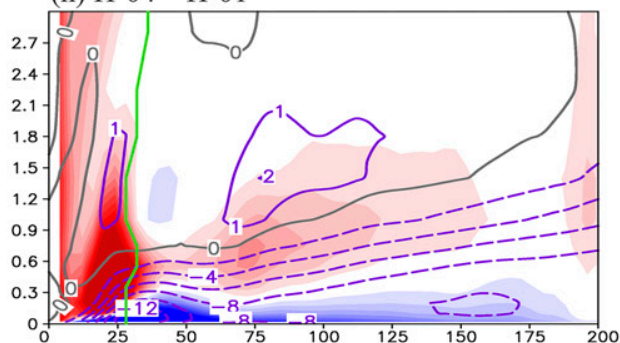

(j) SEF - H06

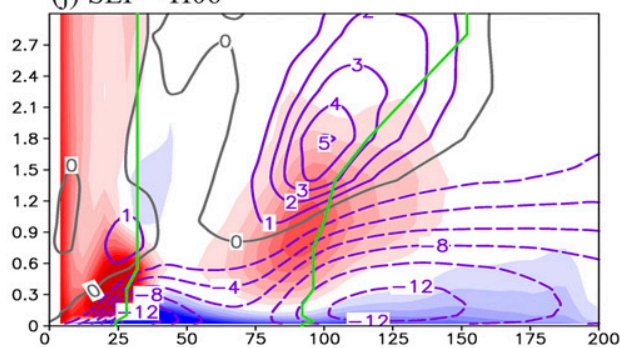

$\left(\mathrm{m} \mathrm{s}^{-1} \mathrm{~h}^{-1}\right)$

FIG. 2. Radius-height cross sections of the temporally averaged mAF [Eq. (4); red: supergradient; blue: subgradient; $\mathrm{m} \mathrm{s}^{-1} \mathrm{~h}^{-1}$ ] superposed with the azimuthal-mean radial velocity [purple; contour interval: $2 \mathrm{~m} \mathrm{~s}^{-1}$ for inflow (dashed), and $1 \mathrm{~m} \mathrm{~s}^{-1}$ for outflow (solid)]. The green line(s) highlights the radius of the local maximum in the azimuthal-mean tangential wind. (a)-(i) Results before SEF. (j) The concentric eyewalls. Hours relative to the SEF time are labeled in the title of each subplot. 
where the secondary supergradient force patch persistently develops (Fig. 2j).

Figure 3 presents the radial profiles of the azimuthal mean tangential, gradient, and agradient winds averaged between $0.5-$ and $1.5-\mathrm{km}$ altitude, the heights for which the supergradient forces are prevalent (Fig. 2) and the secondary tangential jet maximizes (Fig. 1a). Results are shown after the supergradient forces becomes a robust feature in the SEF region. The gradient wind relationship in the axisymmetric point of view and the corresponding agradient wind are defined as follows:

$$
\begin{gathered}
-\frac{1}{\bar{\rho}} \frac{\partial \bar{P}}{\partial r}+f \overline{v_{g}}+\frac{\bar{v}_{g}^{2}}{r}=0 \quad \text { and } \\
\overline{v_{a}}=\bar{v}-\bar{v}_{g},
\end{gathered}
$$

where $\overline{v_{g}}$ is the azimuthal-mean gradient wind and $\overline{v_{a}}$ is the azimuthal-mean agradient wind. About $13 \mathrm{~h}$ prior to $\mathrm{SEF}$, the depth-averaged tangential winds are mostly in gradient wind balance (Figs. 3a,b). When the outer wind field further expands and the rate of the radial decay of tangential winds reduces between 75 and $150 \mathrm{~km}$, the supergradient winds now explain about $10 \%$ of the total azimuthal-mean tangential winds in the same radial interval (Figs. 3c-f). During and after SEF, the increase of tangential wind concentrated in the SEF region (about 4-6 $\mathrm{m} \mathrm{s}^{-1}$ ) is largely attributed to the agradient wind component (Figs. 3g,h), which is around $10 \%-15 \%$ of the total azimuthal-mean tangential wind in that same region. The lighter-colored curves show small variances in the total tangential velocity (gray) in each selected time span outside the eyewall, particularly in the SEF region. The gradient (green) and agradient (pink and blue) components, separately, carry more noticeable temporal variance while still showing qualitative consistency with their temporal mean.

Figure 4 presents the evolving mean tendency of the azimuthal-mean tangential and agradient winds in the SEF region. It shows that, between $\mathrm{H}-07$ and $\mathrm{H}-01$, about two-thirds of the abrupt increase in the positive tangential wind tendency below $2 \mathrm{~km}$ (the major tangential jet region as shown in Fig. 1a) is attributed to the agradient wind tendency (green lines in Fig. 4). This diagnostic calculation suggests that dynamical processes responsible for the reduced subgradient winds in the near surface and the increased supergradient winds in the rest of the boundary inflow layer and up to $2 \mathrm{~km}$ lie at the heart of the SEF problem. Further inferences from the tangential wind tendency equation are presented in the next section.

\section{b. Sharpening and strengthening of boundary layer convergence}

The temporal evolution of the radius-height cross section of the azimuthal-mean divergence is summarized in Fig. 4 of HMW12. To further investigate how the boundary layer horizontal convergence develops in relation to the evolving radial flow, we show here the evolution of the mean radial gradient of radial velocity $\partial \bar{u} / \partial r$ and $\bar{u} / r$ (Figs. 5a,b) in the SEF region. The sum of these two quantities forms the azimuthally averaged horizontal divergence $(\bar{\delta}=\partial \bar{u} / \partial r+\bar{u} / r$; Fig. $5 \mathrm{c})$. Within and just above the boundary inflow layer, the mean radial gradient of radial velocity monotonically becomes more negative (i.e., more convergent) prior to SEF (Fig. 5a). While the radial gradient of inflow persistently sharpens, the changes in $\bar{u} / r$ are relatively small and more subtle (Fig. 5b). ${ }^{3}$ This demonstrates that the growth of boundary layer convergence in the SEF region is largely attributed to the sharpening of the mean radial gradient of inflow. Furthermore, the secondary region of maximum supergradient forces is located in the region where the radial gradient of the boundary layer inflow sharpens (Figs. 2e-h). This calculation supports the hypothesis articulated by HMW12 that the positive agradient force in association with relatively strong supergradient winds acts to arrest the inflowing air parcels outside of the primary eyewall, which, in turn, causes an enhancement of boundary layer convergence and an eruption of air out of the boundary layer. To provide a more complete picture of the boundary layer dynamics of the simulated SEF region (Fig. 1), the upcoming section analyzes the tendency of mean tangential velocity and radial velocity.

\section{Results of momentum budget analyses}

\section{a. Tangential velocity tendency equation}

Dynamical processes contributing to the establishment of the secondary maximum in the azimuthal-mean tangential wind in the boundary layer and the lower troposphere (Fig. 1a) may be quantitatively investigated using a diagnosis of the tangential velocity tendency equation shown in Eq. (1). Figures 6-10 show diagnostic analyses at three selective time windows prior to SEF. Figure 6 a demonstrates the intensification of the original eyewall and the expanding wind field outside the radius of $75 \mathrm{~km}$ and below the altitude of $5 \mathrm{~km}$ during H-19 and H-13 (see also the evolution illustrated in Figs. 3a,b).

\footnotetext{
${ }^{3}$ The insignificant change of the area mean $\bar{u} / r$ does not conflict with the fact that the area-mean inflow in the SEF region slightly strengthens prior to SEF (Fig. 5a of HMW12). As inflowing air considerably decelerates across the SEF region, stronger inflow is present at larger radii, and weaker inflow is present at smaller radii. The influence of the enhanced inflow on the increment of $\bar{u} / r$ is lessened by the larger radii. This explains why an increase in the area mean inflow can yield a subtle change in the areal mean of $\bar{u} / r$.
} 
(a) H-19-H-16

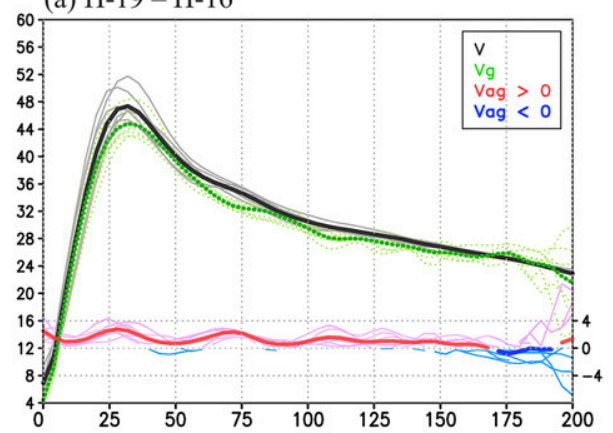

(c) $\mathrm{H}-13-\mathrm{H}-10$

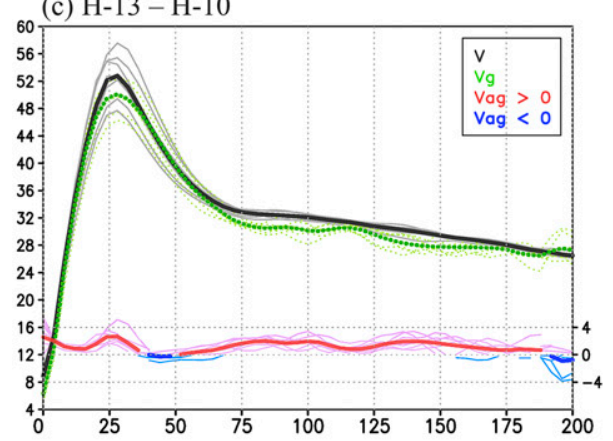

(e) $\mathrm{H}-07-\mathrm{H}-04$

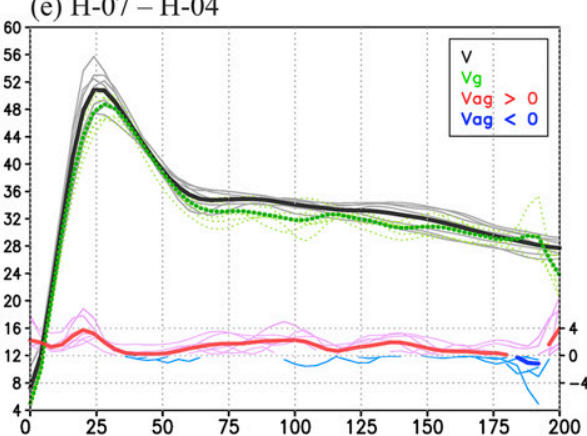

(g) H-01 - SEF

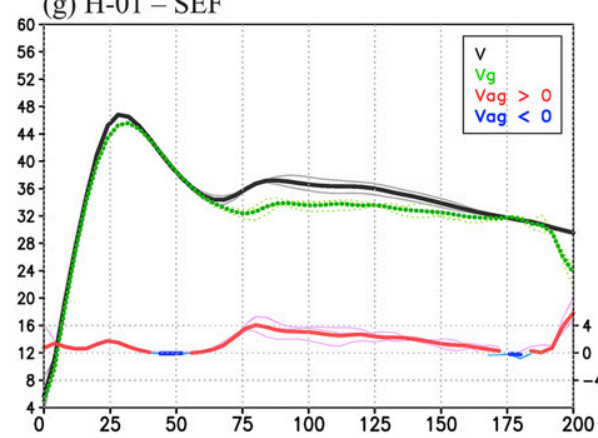

(b) $\mathrm{H}-16-\mathrm{H}-13$

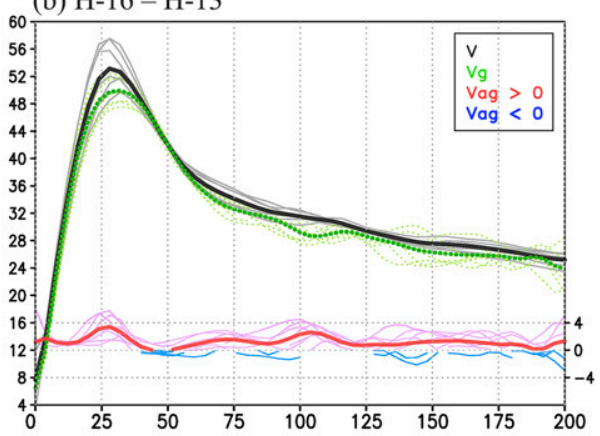

(d) $\mathrm{H}-10-\mathrm{H}-07$

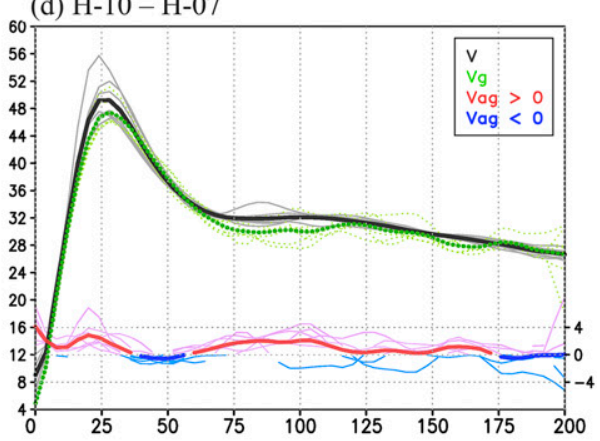

(f) $\mathrm{H}-04-\mathrm{H}-01$

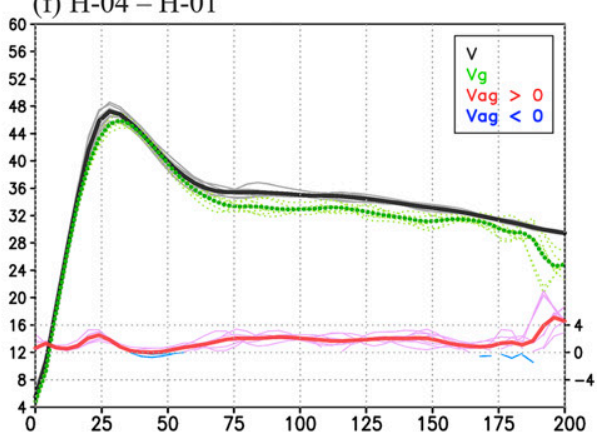

(h) SEF - H06

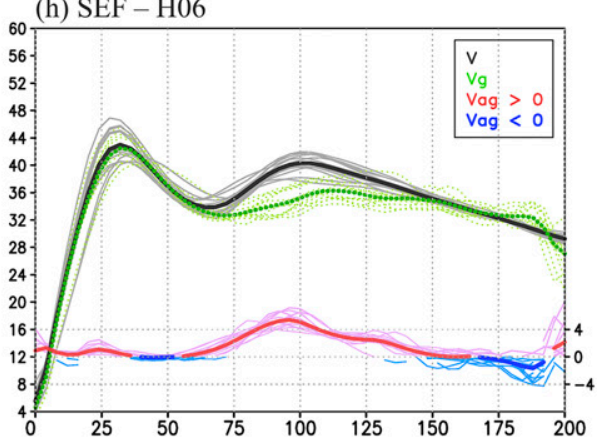

FIG. 3. Radial profile of various azimuthally averaged tangential wind quantities $\left(\mathrm{m} \mathrm{s}^{-1}\right)$. (a)-(g) Results before SEF, sharing the same time windows as Figs. 2c-i. (h) The concentric eyewall structure in the same time window as Fig. 2j. Results are shown every $30 \mathrm{~min}$ (thin lines). The mean tangential velocity quantities are vertically averaged also between 0.5 - and $1.5-\mathrm{km}$ height, the height interval wherein supergradient winds are prevalent in the eyewall regions. The azimuthal-mean tangential wind $v$ and gradient wind $v_{g}$ are shown in gray and green, respectively; $v_{\mathrm{ag}}$ is the azimuthal-mean agradient wind $\left(v_{\mathrm{ag}}=v-v_{g}\right)$. The supergradient $\left(v_{\mathrm{ag}}>0\right)$ and subgradient $\left(v_{\mathrm{ag}}<0\right)$ winds are in magenta and blue, respectively. The temporal averages in the corresponding time window are in bold. The scale of $v_{\text {ag }}$ is labeled at the right $y$ axis. 
(a) $\partial \bar{v} / \partial t$

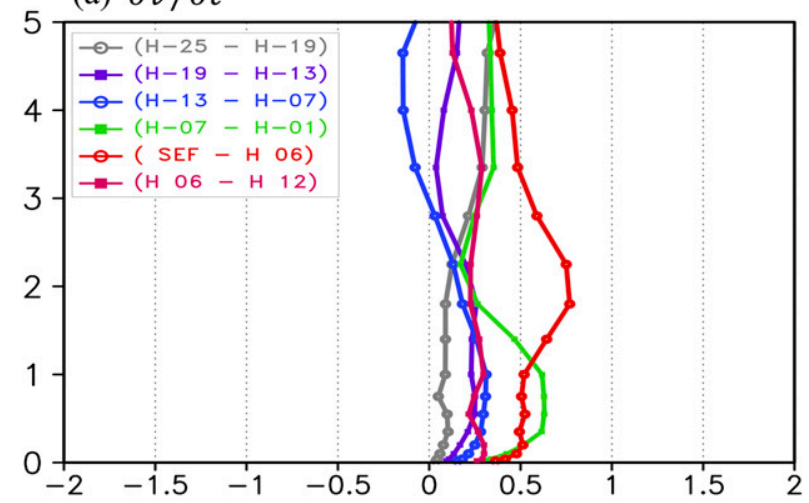

(b) $\partial \overline{v_{a g}} / \partial t$

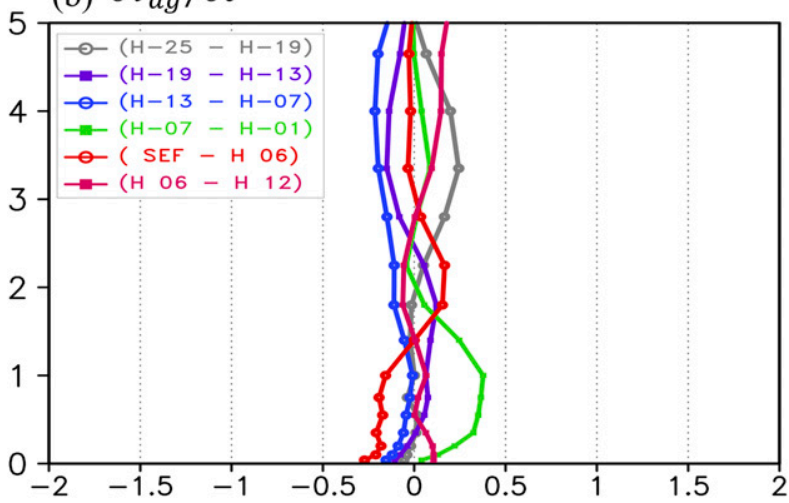

FIG. 4. (a) The simulated tendency of the azimuthal-mean tangential velocity and (b) corresponding agradient tangential velocity tendency $\left(\mathrm{m} \mathrm{s}^{-1} \mathrm{~h}^{-1}\right)$, averaged in the SEF region $(r=75-125 \mathrm{~km})$ over the indicated time windows. The ordinate denotes height $z(\mathrm{~km})$ above ocean surface.

Positive tendencies in the vortex's outer core maximize at around $2 \mathrm{~km}$ in the SEF region and below $1 \mathrm{~km}$ outside of the SEF region. Negative tangential wind tendencies are located at radii between 50 and $75 \mathrm{~km}$, which is the moat region (Fig. 1a). From H-13 to H-07, the original eyewall starts to weaken, and the increase of the tangential wind in the vortex's outer core concentrates in the SEF region and below $3 \mathrm{~km}$ (Fig. 6b; cf. Figs. $3 \mathrm{c}$ and $3 d$ ). Subsequently, the original eyewall continues to weaken, and the positive tendency in the outer-core region doubles (as seen by examining the $0.5 \mathrm{~m} \mathrm{~s}^{-1} \mathrm{~h}^{-1}$ contour in Fig. 6c) from H-07 to H-01 (cf. Figs. 3e and 3f). The region of the greatest tendencies are located within or slightly above the top of the boundary layer in the SEF region. The vertical extent of the positive tendency region increases with increasing radii.

Figures 7-9 present the impact of the individual terms on the right-hand side of Eq. (1) on the local tendency of the azimuthal-mean tangential winds. Within the boundary inflow layer, $\bar{F}_{\lambda}$ is the largest term to diminish (a) $\partial \bar{u} / \partial r$

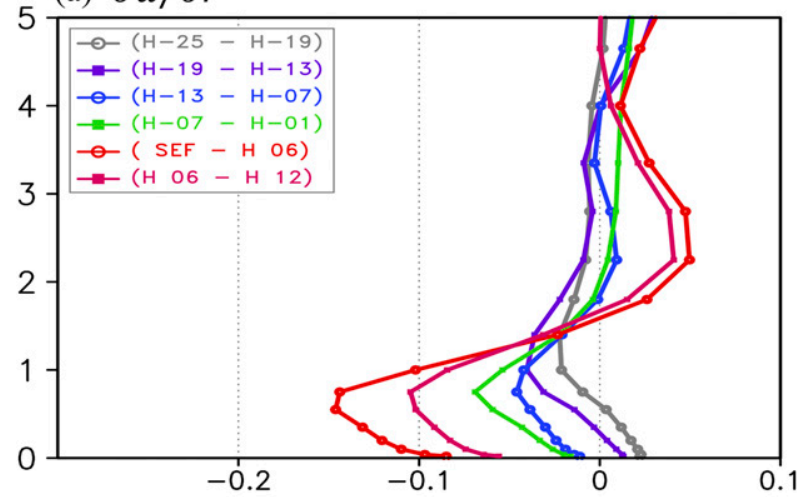

(b) $\bar{u} / r$

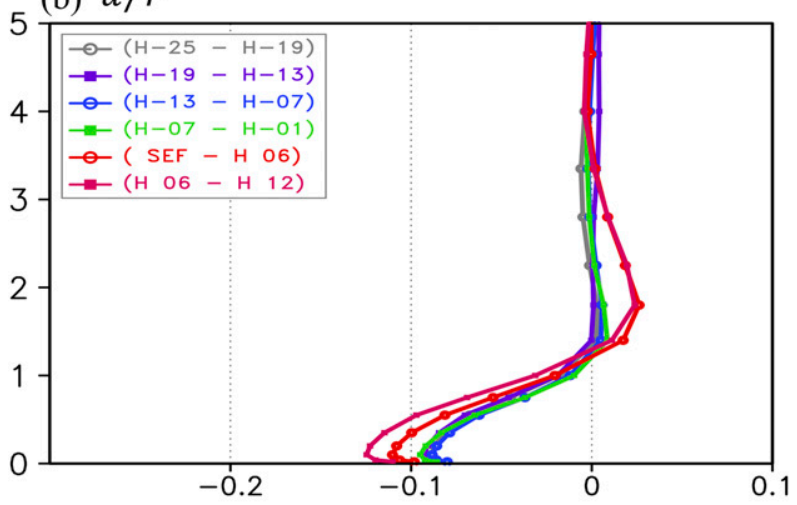

(c) $\partial \bar{u} / \partial r+\bar{u} / r$

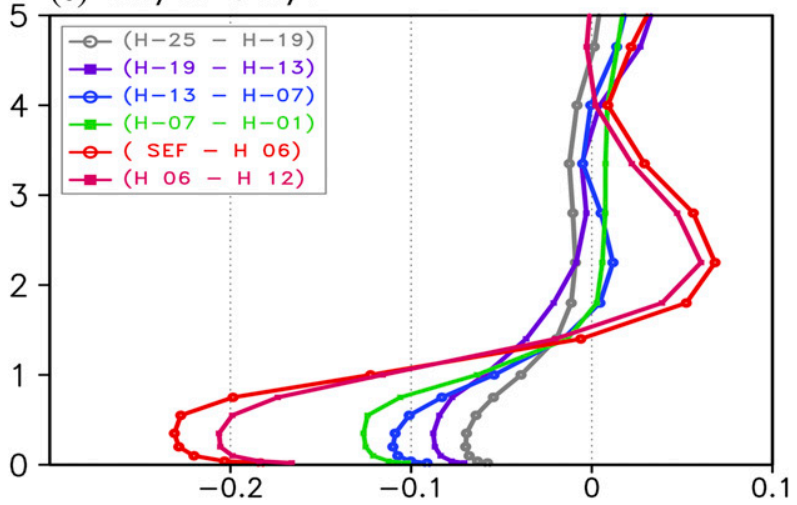

FIG. 5. As in Fig. 4, but for (a) the radial gradient of the azimuthalmean radial velocity and (b) corresponding radial velocity divided by radius; (a) together with (b) is (c) the azimuthal-mean horizontal divergence. Units for all panels are $\mathrm{s}^{-1} \times 10^{-3}$.

the tangential winds (Figs. 7a,c,e), and the radial fluxes of absolute vorticity due to the mean flow are the largest terms to enhance the tangential winds (Figs. $7 \mathrm{~b}, \mathrm{~d}, \mathrm{f}$ ). It is apparent that the gain from the latter substantially compensates the loss due to $\bar{F}_{\lambda}$. Because $\bar{w}$ is mostly positive, the mean component of vertical advection contributes positively above the height of the maximum tangential winds, which is located slightly within the boundary inflow layer (Figs. 8a,c,e). The impact of eddy 
(a) $\mathrm{H}-19 \sim \mathrm{H}-13$

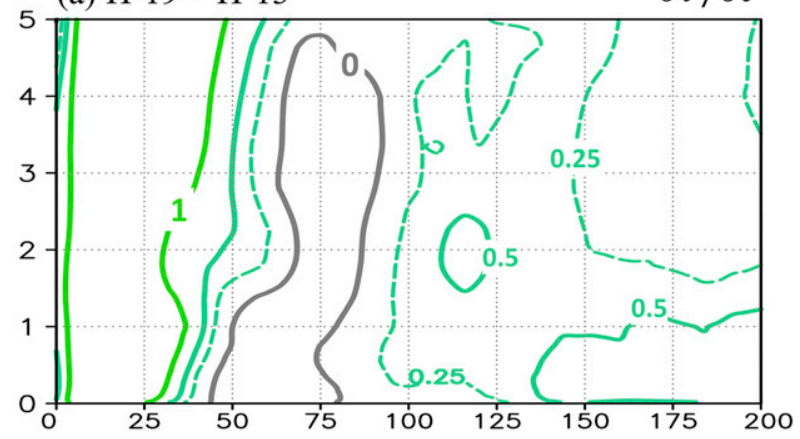

(b) $\mathrm{H}-13 \sim \mathrm{H}-07$

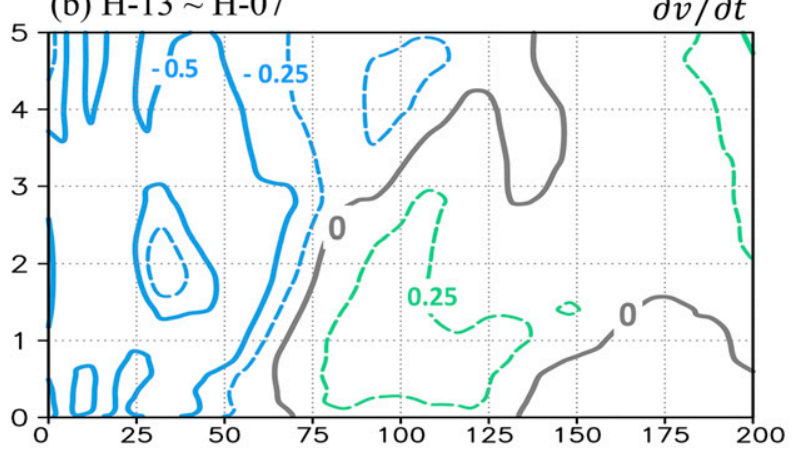

(c) $\mathrm{H}-07 \sim \mathrm{H}-01$

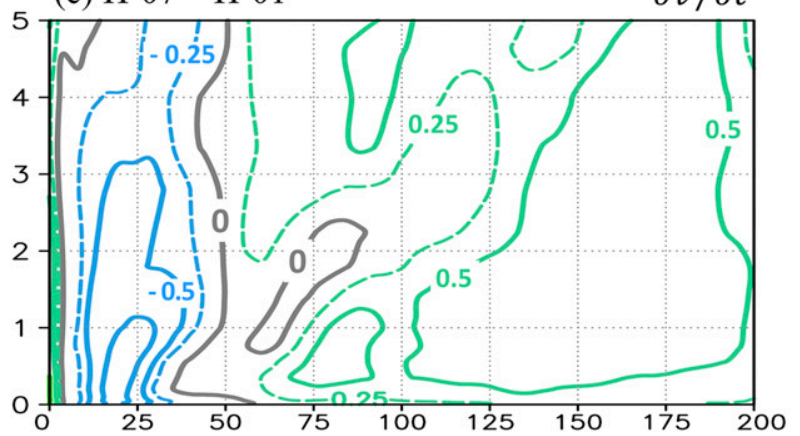

FIG. 6. Radius-height cross sections of the simulated local azimuthal-mean tangential wind tendency averaged between (a) H-19 and H-13, (b) $\mathrm{H}-13$ and $\mathrm{H}-07$, and (c) $\mathrm{H}-07$ and $\mathrm{H}-01$. Solid contours are $-1,-0.5,0,0.5$, and $1 \mathrm{~m} \mathrm{~s}^{-1} \mathrm{~h}^{-1}$. Additional contours of $\pm 0.25 \mathrm{~m} \mathrm{~s}^{-1} \mathrm{~h}^{-1}$ are added for clarity. Here, positive tendencies are green, and negative tendencies are blue. The zero-tendency contour is gray.

vertical advection is similar but is of a smaller magnitude and vertical and radial extent (Figs. 8b,d,f). Finally, Fig. 9 shows the radial flux of absolute vorticity due to eddies. The positive contribution is confined to the moat region and inner side of the SEF region. The positive impact on the inner side of the SEF region, present at the lowest 1 or $2 \mathrm{~km}$, starts to become limited about half a day prior to SEF (Figs. 9b,c).

Table 1 summarizes previous research that conducted a horizontal momentum budget analysis for storms undergoing SEF. Here, we make comparisons with those studies focused on the time period prior to SEF. The two azimuthal-mean terms and the effect due to the subgrid-scale stress divergence terms in the PBL are qualitatively consistent with analyses conducted in previous studies, such as Rozoff et al. (2012), Sun et al. (2013), Abarca et al. (2015), and Wang et al. (2016). The results of two azimuthal eddy terms are divergent among different studies. Our calculations of the eddy contributions are fairly consistent with those in Rozoff et al. (2012) and Sun et al. (2013). These two studies both presented the WRF simulated results at a finer horizontal grid spacing and with more vertical layers. The former carried out an experiment initialized with an axisymmetric cyclonic vortex and integrated on a $\beta$ plane, while the latter performed a simulation of Sinlaku. The eddy contributions in our calculation are different from Abarca et al. (2015) and Wang et al. (2016). Presenting the areal-mean results in the SEF region, Abarca et al. (2015) demonstrated the small positive contribution of eddy radial vorticity fluxes within and just above the boundary inflow layer, and Wang et al. (2016) showed this positive contribution occupies a broad area outside the eyewall. While the eddy vertical advection in the SEF region presented in Abarca et al. (2015) is similar to our calculation, Wang et al. (2016) obtained a sandwich-like pattern that has a negative impact at around $1-2-\mathrm{km}$ altitude above the sea surface. The calculations in Abarca et al. (2015) and Wang et al. (2016) are based on idealized simulations on an $f$ plane and with no background vertical wind shear. An idealized configuration possessing neither background vorticity gradient nor vertical wind shear very likely produces a vortex without a persistent wavenumber-1 structure (e.g., Judt and Chen 2010; Fang and Zhang 2012). Such a configuration is expected to be devoid of a principal rainband (e.g., Willoughby et al. 1984; Houze 2010) as observed in many realistic tropical cyclones that undergo SEF [e.g., Hurricane Rita (2005) and Typhoon Sinlaku (2008)]. Wang et al. (2016) provided analyses to show that higher wavenumber asymmetries account for the positive contribution of the eddy vorticity flux within the boundary layer in their simulation. On the basis of this prior work, it seems plausible that the role of eddies in the tangential wind tendency, and in SEF, is different when the effects of the background vorticity gradient and vertical wind shear are excluded.

The foregoing time-evolving flow characteristics can be succinctly synthesized using radially averaged quantities in the SEF region. From the point of view of the areal mean, we examine the temporal evolution of the azimuthal-mean tangential wind tendency in the SEF region for a shorter time interval. Consistent with the 
(a) $\mathrm{H}-19 \sim \mathrm{H}-13$

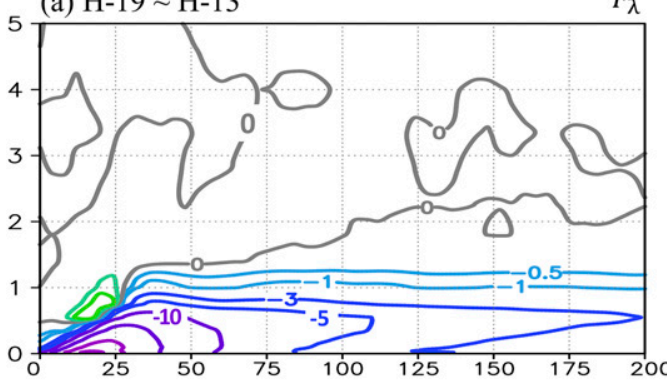

(c) $\mathrm{H}-13 \sim \mathrm{H}-07$

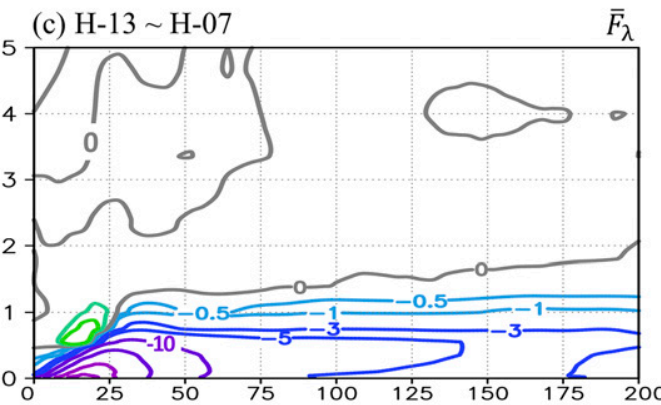

(e) $\mathrm{H}-07 \sim \mathrm{H}-01$

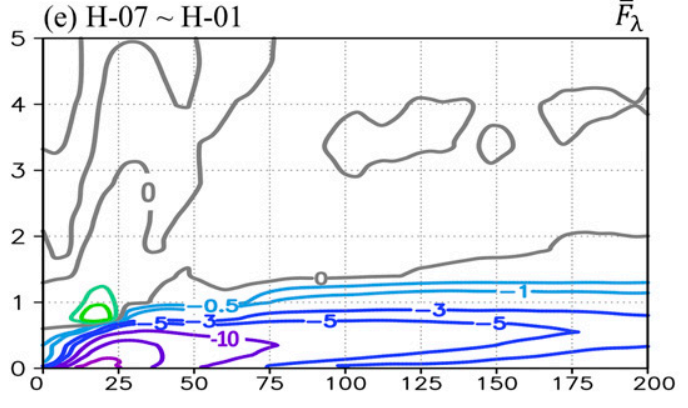

(b) $\mathrm{H}-19 \sim \mathrm{H}-13$

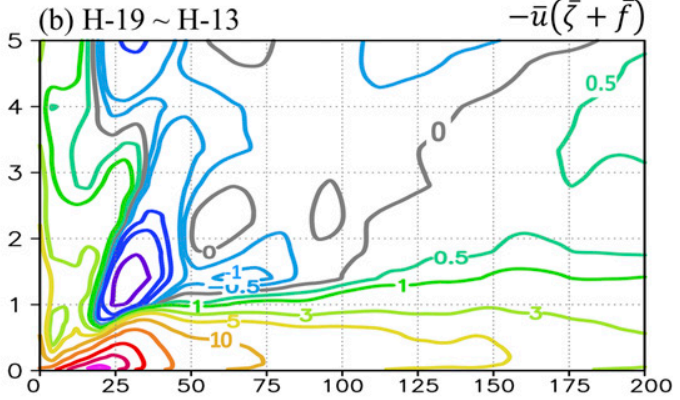

(d) $\mathrm{H}-13 \sim \mathrm{H}-07$

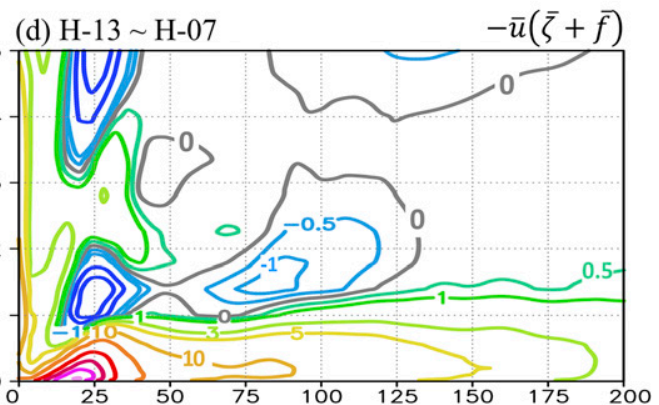

Contours: $-60,-40,-20,-10,-5,-3,-1,-0.5,0,0.5,1,3,5,10,20,40,60,80,100 \quad\left(\mathrm{~m} \mathrm{~s}^{-1} \mathrm{~h}^{-1}\right)$

FIG. 7. Radius-height cross sections of temporally averaged quantities. (a),(c),(e) The azimuthal-mean tangential wind tendency due to the PBL and surface schemes. (b),(d),(f) The tendency associated with the mean radial flux of absolute vorticity. Positive tendencies are green and warm colors, while negative tendencies are blue and purple. The zero-tendency contour is gray. The magnitude and sign of tendency are below the plots. The time window (integration hours relative to the SEF time) is indicated in the title of each subplot.

discussion on Figs. 7-9, in the SEF region, the mean radial flux of absolute vorticity and the tangential wind tendency due to the PBL parameterization have the greatest absolute magnitude (Fig. 10). Within the boundary inflow layer, the mean radial flux of absolute vorticity contributes to the increase of the tangential wind tendency, while the effect due to the PBL parameterization acts to weaken the tangential wind (Figs. 10a,b). It is apparent that the positive contribution from the mean radial flux of absolute vorticity always outweighs the negative effect of the PBL parameterization on the tangential wind tendency. Individual impacts of both the mean radial flux of absolute vorticity and the PBL parameterization become stronger with time either before or after SEF. While the effect due to the PBL parameterization steadily and slightly increases with time, the monotonic increase of the mean radial flux of absolute vorticity becomes greater at around $\mathrm{H}-12$ and has a jump upon SEF. The greater increase of $-\bar{u} \bar{\zeta}_{a}$ at around H-12 implies also the increasing dominance of $-\bar{u} \overline{\zeta_{a}}$ in the evolving swirling winds in the SEF region after H-12. About half a day before SEF, the mean vertical advection yields small but persistent positive impacts on the swirling wind at around 1-2-km height, assisting the expansion of the outer wind field above the boundary layer (gray, blue, and purple lines in Fig. 10d). The eddy radial vorticity flux starts to slightly and negatively affect the tangential wind in the lowest $3 \mathrm{~km}$ about $6 \mathrm{~h}$ prior to SEF (green lines in Fig. 10c). Meanwhile, the positive contribution of the mean vertical advection becomes greater and the eddy vertical advection starts to 
(a) $\mathrm{H}-19 \sim \mathrm{H}-13$

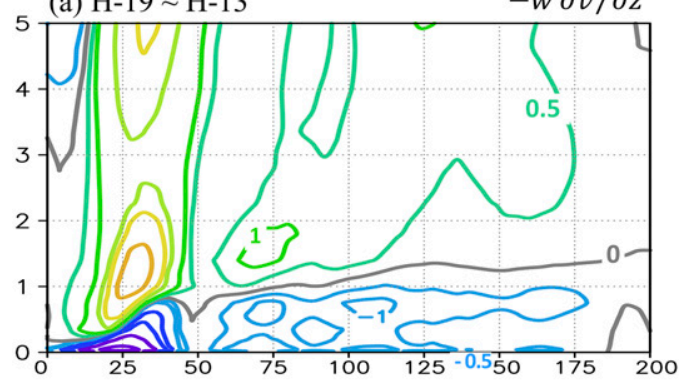

(c) $\mathrm{H}-13 \sim \mathrm{H}-07$

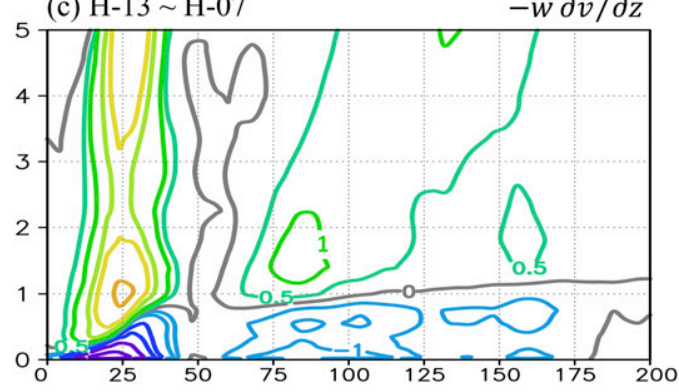

(e) $\mathrm{H}-07 \sim \mathrm{H}-01$

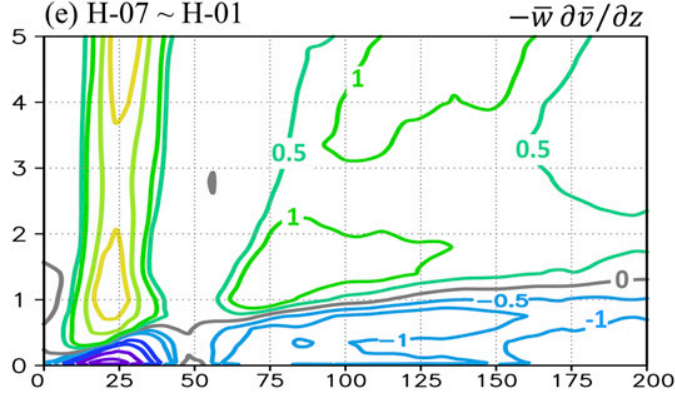

(b) $\mathrm{H}-19 \sim \mathrm{H}-13$

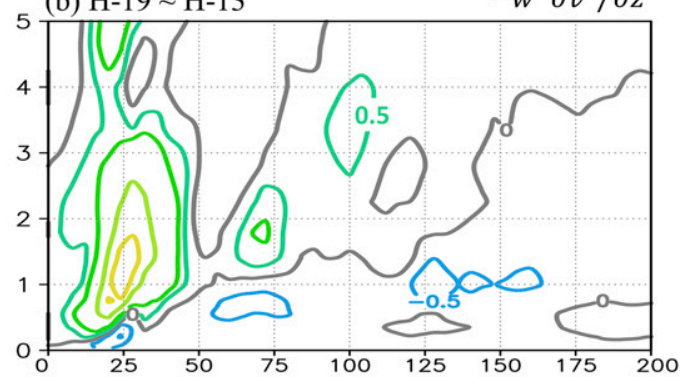

(d) $\mathrm{H}-13 \sim \mathrm{H}-07$

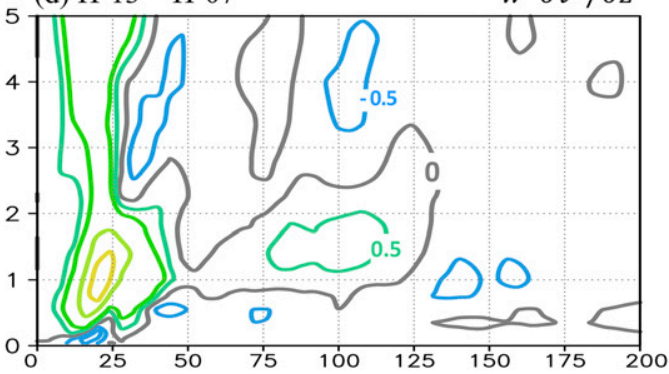

(f) $\mathrm{H}-07 \sim \mathrm{H}-01 \quad-\overline{w^{\prime} \partial v^{\prime} / \partial z}$

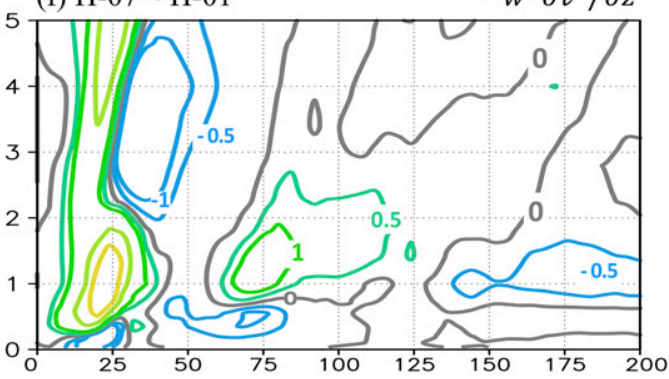

Contours: $-60,-40,-20,-10,-5,-3,-1,-0.5,0,0.5,1,3,5,10,20,40,60,80,100\left(\mathrm{~m} \mathrm{~s}^{-1} \mathrm{~h}^{-1}\right)$

FIG. 8. As in Fig. 7, but showing the azimuthal-mean tangential wind tendency associated with the (a),(c),(e) mean and (b),(d),(f) eddy components of the azimuthal-mean vertical advections.

show clear but small positive impacts at heights around $1-2 \mathrm{~km}$ (green lines in Figs. 10d,e). Within the boundary layer, vertical advection due to the mean flow has a relatively small negative impact prior to SEF, and the influence of eddy vertical advection is negligible.

The foregoing analysis of the tangential velocity tendency equation suggests that the competition between the mean radial flux of absolute vorticity and the effect due to the PBL parameterization, in particular the more pronounced increase in the mean radial flux of absolute vorticity, is key to the progressive increase in the azimuthal-mean tangential wind in the boundary inflow layer in the SEF region before SEF. The temporal increase of the tangential winds in the boundary layer leading up to SEF is confirmation of the importance of the boundary layer spinup mechanism when time dependence is included. The vertical advection, including its mean and eddy components, leads to the vertically upward extension of the expanding swirling circulation.

\section{b. Radial velocity tendency equation}

To provide a more complete picture of the dynamics behind the rapid deceleration of the radial inflow in the boundary layer in the simulated SEF region, we analyze now the material derivative of the mean radial wind. Different from the Eulerian viewpoint previously applied to the tangential velocity, the Lagrangian perspective permits one to precisely identify how the boundary layer inflow is decelerated when passing through the SEF region, where a prominent radial gradient of the azimuthal-mean radial flow and the associated boundary layer convergence are established prior to SEF (Figs. 1 and 5). The agradient force HMW12 examined is the mAF shown in Eq. (4). In this study, 


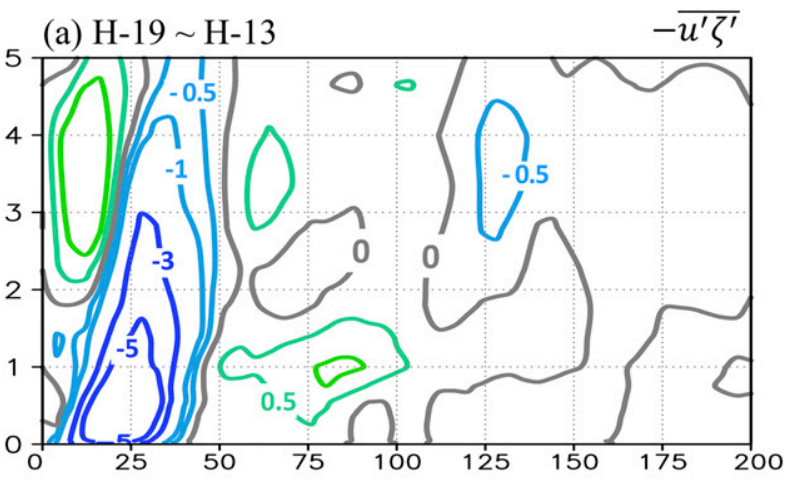

(b) $\mathrm{H}-13 \sim \mathrm{H}-07$

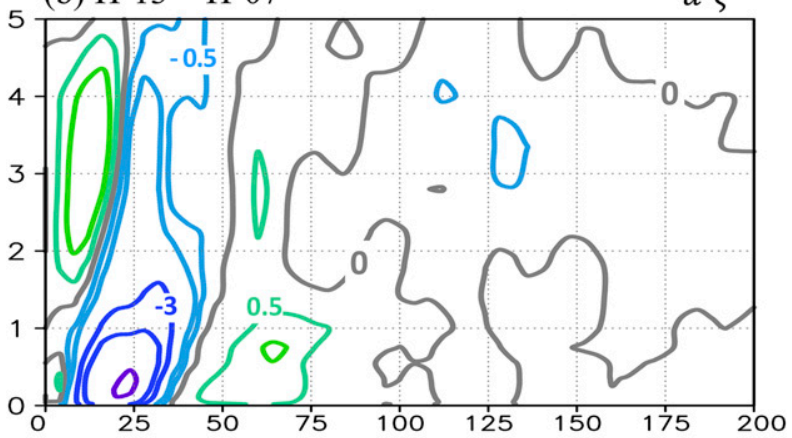

(c) $\mathrm{H}-07 \sim \mathrm{H}-01$

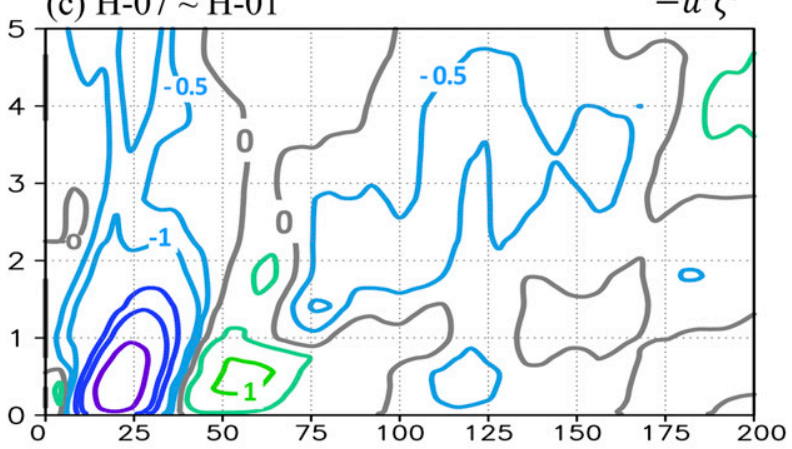

Contours: $-60,-40,-20,-10,-5,-3,-1,-0.5,0$,

$$
0.5,1,3,5,10,20,40,60,80,100\left(\mathrm{~m} \mathrm{~s}^{-1} \mathrm{~h}^{-1}\right)
$$

FIG. 9. As in Fig. 7, but showing the azimuthal-mean tangential wind tendency due to the eddy radial flux of absolute vorticity.

a complete budget calculation of material derivative of the azimuthal-mean radial wind [see Eqs. (2)-(5)] is presented. In addition to $\mathrm{mAF}$, the eAF [described in Eq. (5)] and the effect due to PBL and surface parameterizations $\overline{F_{r}}$ are investigated. Figure 11 presents the diagnosed results in the SEF region. The maximum of $\mathrm{mAF}$ is an order greater than that of eAF (cf. Figs. 11a and 11b), and the eddy pressure gradient force $\left[-\overline{(1 / \rho)\left(\partial P^{\prime} / \partial r\right)}\right]$ is an order smaller than the eddy centrifugal force $\left(v^{\prime 2} / r\right)$ (figure not shown), consistent with calculations of Abarca et al. (2015). Figure 11c shows that $\langle\mathrm{AF}\rangle$, which includes both the eddy and mean contributions, presents a slightly more pronounced increasing tendency of supergradient forces than mAF prior to SEF. Because of the inconsequential difference between $\mathrm{mAF}$ and $\langle\mathrm{AF}\rangle$, the conclusion drawn in HMW12 (based on $\mathrm{mAF}$ ) remains valid-namely, the progressive and monotonic increase of the supergradient forces starting about 1 day before SEF.

To understand how the inflow decelerates when passing through the SEF region and how the boundary layer convergence strengthens, the present discussion is first concentrated on the boundary inflow layer. The value of $\overline{F_{r}}$ is positive in the boundary inflow layer and pronouncedly decelerates the inflowing air parcels near the surface (Fig. 11d). The contribution of the effect due to PBL and surface parameterization rapidly diminishes with increasing height in the boundary inflow layer. In contrast to $\overline{F_{r}}$, the agradient force, either $\langle\mathrm{AF}\rangle$ or $\mathrm{mAF}$, is negative (subgradient) near the surface. The agradient force becomes positive (supergradient) a few hundreds of meters above the surface (Figs. 11a,c). In the upper portion of the inflow layer, the supergradient force together with $\overline{F_{r}}$ decelerate the inflowing air, consistent with results presented by Abarca et al. (2015). At these altitudes, the magnitude of supergradient forces increases with increasing height and peaks at around 1-km height (Fig. 11c), while the magnitude of $\overline{F_{r}}$ diminishes with increasing height and vanishes at the altitude of $<1 \mathrm{~km}$ (Fig. 11d). Furthermore, during the 1-day period preceding SEF, supergradient forces progressively and monotonically increase and extend downward in the SEF region, while the profile of $\overline{F_{r}}$ barely varies (cf. Figs. 11c and 11d). Therefore, changes of the net radial force (Fig. 11f) are primarily attributed to the changes in $\mathrm{mAF}$.

The competing tendencies between $\overline{F_{r}}$ and $\langle\mathrm{AF}\rangle$ determine whether the inflowing air is decelerated or accelerated across the SEF region. Figure 11f demonstrates that the negative (radially inward) net force acts to accelerate the boundary layer inflowing parcels that pass through the SEF region before $\mathrm{H}-21$ (the two gray lines) when the original eyewall is intensifying. This may correspond to the boundary layer spinup mechanism as articulated by Smith and Montgomery (2015). Later on, because of the increasing and downward-extending positive agradient forces, the net effect of $\overline{F_{r}}$ and $\langle\mathrm{AF}\rangle$ becomes positive (radially outward), rapidly decelerating boundary inflow air across the SEF region after H-18. This positive net force in the radial direction monotonically increases in time and exhibits a near jump around the SEF time. The increase in this positive net force continues for a few hours after SEF (orange) and 
(a) $\bar{F}_{\lambda}$

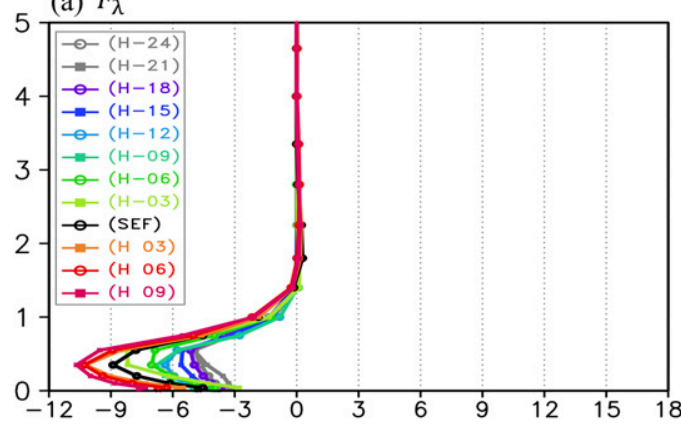

(c) $-\overline{u^{\prime} \zeta^{\prime}}$

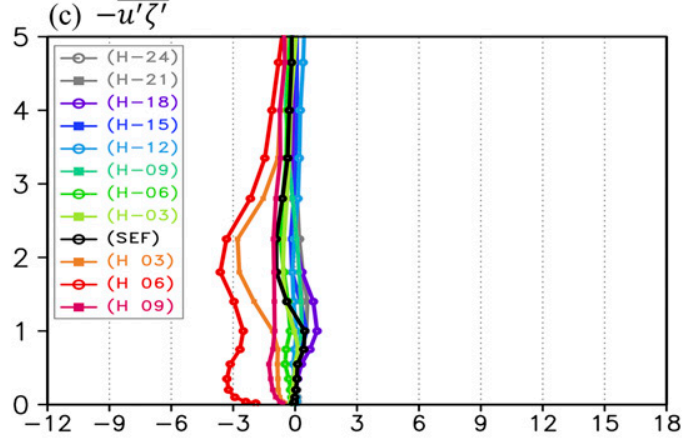

(b) $-\bar{u}(\bar{\zeta}+\bar{f})$

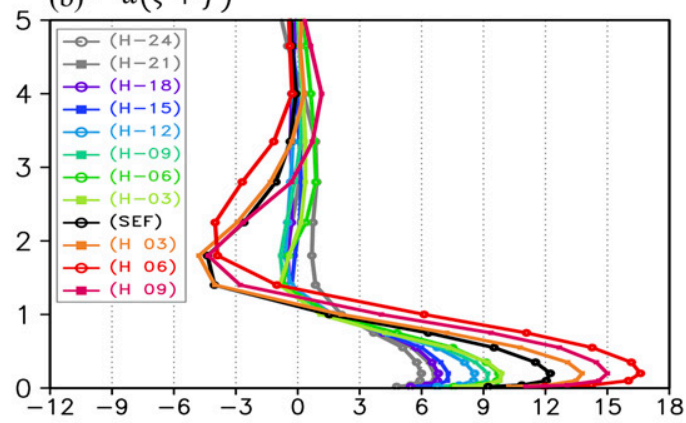

(d) $-\bar{w} \partial \bar{v} / \partial z$

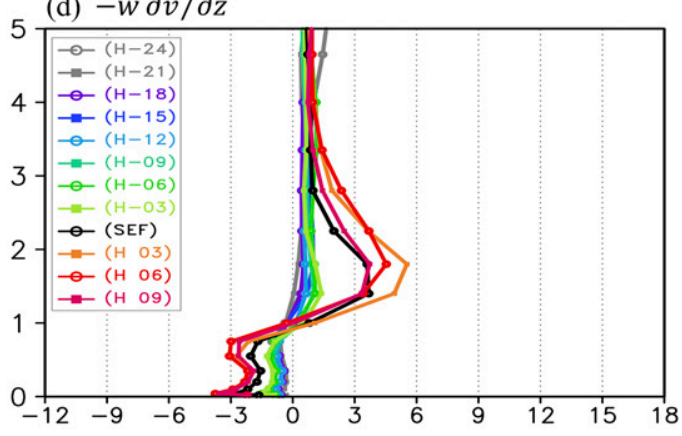

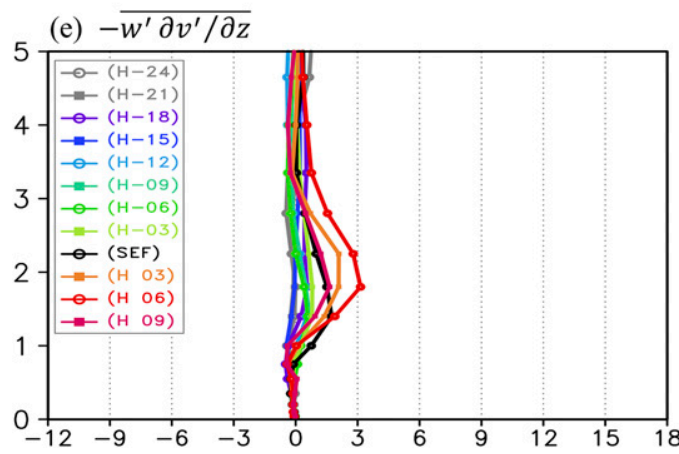

FIG. 10. Diagnosed results of the tendency equation of the azimuthal-mean tangential velocity [Eq. (1)]. The abscissa presents the tendency $\left(\mathrm{m} \mathrm{s}^{-1} \mathrm{~h}^{-1}\right)$ averaged over a time window between $t-1.5 \mathrm{~h}$ and $t+1.5 \mathrm{~h}$ and over the SEF region $(r=75-125 \mathrm{~km})$. Here, $t$ is given in the legend, showing the hours relative to the SEF time. The ordinate denotes the height (km). The azimuthal-mean tangential wind tendency associated with (a) effects of the PBL and surface parameterizations, (b) mean and (c) eddy radial fluxes of absolute vorticity, and (d) mean and (e) eddy vertical advection.

weakens afterward (red). It is noted again that the increase in the positive agradient force is marked prior to $\mathrm{SEF}$, while changes in $\bar{F}_{r}$ are minimal. This result implies that the changes in $\langle\mathrm{AF}\rangle$ principally explain the evolving net radial force.

The foregoing analysis of the radial wind acceleration provides new confirmational evidence for the SEF pathway proposed in HMW12. In particular, the intensifying agradient force is responsible for the sharpening of the boundary layer inflow in the SEF region. Such persistent forcing contributes to the buildup of boundary layer convergence, which, in turn, forces moist boundary layer parcels to rise into the free atmosphere and promote a secondary deep convection region outside of the primary eyewall.

\section{Discussion}

\section{a. Tangential wind tendency}

The diagnosed total tendencies of the azimuthal-mean tangential wind [the summation of the terms on the right-hand side of Eq. (1)] end up with very small magnitudes as compared to each individual term, giving rise to potential inaccuracies in the net Eulerian tendency. In this study, we present results in a way to create 
TABLE 1. Studies that carried out calculations of horizontal momentum budgets for storms undergoing SEF. Figure numbers refer to the figures in the corresponding references.

\begin{tabular}{|c|c|c|c|c|}
\hline References & $\begin{array}{l}\text { Tangential momentum } \\
\text { analysis }\end{array}$ & $\begin{array}{l}\text { Forcing terms } \\
\quad \text { of } d \bar{u} / d t\end{array}$ & $\begin{array}{l}\text { Before/after } \\
\text { SEF }\end{array}$ & Data \\
\hline Didlake and Houze (2011) & Fig. 12 & Fig. 6 & After & Observations \\
\hline Rozoff et al. (2012) & Fig. 4 & No & Before & $\begin{array}{l}\text { WRF simulation and Sawyer-Eliassen } \\
\text { diagnosis }\end{array}$ \\
\hline Abarca and Montgomery (2013) & Fig. 5 & Fig. 4 & Before & RAMS simulation \\
\hline Kepert (2013) & Figs. 3,4 & Figs. 3,4 & After & $\begin{array}{l}\text { Diagnosed results of a nonlinear } \\
\text { boundary layer model }\end{array}$ \\
\hline Wang et al. (2013) & Fig. 16 & Figs. $10,11,15$ & Before & MM5 simulation \\
\hline Sun et al. (2013) & Figs. 10, 15 & No & Before & WRF simulation \\
\hline Qiu and Tan (2013) & Figs. 5,14 & Fig. 14 & Before & WRF simulation \\
\hline Abarca et al. (2015) & Fig. 4 & Fig. 4 & Before & RAMS simulation \\
\hline Zhu and Zhu (2014) & Figs. $6,10,12,13$ & No & After & $\begin{array}{l}\text { WRF simulation and Sawyer-Eliassen } \\
\text { diagnosis }\end{array}$ \\
\hline Wang et al. (2016) & Figs. 6,13 & Fig. 5 & Before & $\begin{array}{l}\text { WRF simulation and Sawyer-Eliassen } \\
\text { diagnosis }\end{array}$ \\
\hline
\end{tabular}

less uncertainty. In Fig. 12, we investigate 1) the competing effect of the two largest terms in the boundary inflow layer $\left(-\bar{u} \bar{\zeta}_{a}+\bar{F}_{\lambda}\right)$ and 2$)$ the total vertical advection as the mean and eddy terms show qualitatively similar results. The competing effect between the mean radial flux of absolute vorticity and $\bar{F}_{\lambda}$ (Figs. 12a,c,e) also reflects the competition between the mean radial advection of cyclonic absolute angular momentum and corresponding loss due to frictional torque. ${ }^{4}$ Discussions on the two processes (the left and right panels in Fig. 12) together with the eddy radial vorticity flux (Fig. 6) are helpful in determining how the low-level tangential winds are reduced or maintained in the moat and how they are elevated in the SEF region.

\section{1) IN THE MOAT REGION}

The maintenance (Figs. 6a,c) or decrease (Fig. 6b) in tangential winds in the moat region (Fig. 1) results from the combined influence of different terms on the righthand side of Eq. (1). The mean radial flux of absolute vorticity contributes negatively to the azimuthal-mean tangential wind tendency over the height around $1-2 \mathrm{~km}$ (Figs. $7 \mathrm{~d}, \mathrm{f}$ ), which is the outflow region atop the

\footnotetext{
${ }^{4}$ In $M=(1 / 2) f r^{2}+r v, M$ stands for the absolute momentum per unit mass. When this equation is azimuthally averaged and differentiated partially in relation to time on both sides, one obtains $\partial \bar{M} / \partial t=r \partial \bar{v} / \partial t$. The impact of the surface friction and subgrid diffusion on $\partial \bar{M} / \partial t$ is obtained similarly via the torque $r \bar{F}_{\lambda}$. The radial advection of $\bar{M}$ is expressed as $-\bar{u} \partial \bar{M} / \partial r=-r \bar{u}(f+\bar{v} / r+\partial \bar{v} / \partial r)=$ $-r \bar{u} \bar{\zeta}_{a}$. The competing effect between the mean radial advection of absolute momentum and frictional loss is thus obtained by the sum of the terms $r\left(-\bar{u} \overline{\zeta_{a}}+\bar{F}_{\lambda}\right)$.
}

boundary inflow layer. Outflow transports absolute angular momentum from smaller radii to larger radii and brings the supergradient wind toward the state of gradient wind balance. At these same heights, vertical advection contributes positively to the tangential wind tendency (Figs. 8 and 12b,d,f). In the upper portion of the boundary inflow layer, the competing effect of the mean radial flux of absolute vorticity (positive) and $\bar{F}_{\lambda}$ (negative) acts to reduce the tangential wind tendency (Figs. 12c,e). Vertical advection furnishes a negative influence throughout the majority of the boundary layer (Figs. 8 and 12b,d,f). Above $2 \mathrm{~km}$, eddy vertical advection starts to have negative impacts on the outer portion of the original eyewall about half a day prior to SEF (Fig. 8d). Later on, the negative influence radially expands toward the moat region (Fig. 8f). These negative impacts shown in Fig. 12 are compensated by the eddy radial flux of absolute vorticity, which has a positive influence on the tangential wind throughout the majority of the moat region (cf. Figs. 9 and 12).

\section{2) IN THE SEF REGION}

From $\mathrm{H}-19$ to $\mathrm{H}-13$, the competing impacts between the terms $-\bar{u} \bar{\zeta}_{a}$ and $\bar{F}_{\lambda}$ act to support the broadening of tangential winds outside the radius of $100 \mathrm{~km}$ throughout the lower troposphere (cf. Figs. 6a and 12a). The net effect produces a weak and near-surface secondary tangential wind maximum around the radius of $75 \mathrm{~km}$ (Fig. 12a). The net effect of these two leading terms in the boundary inflow layer starts to establish a distinct secondary maximum concentrating in the SEF region, within and just above the lowest $1 \mathrm{~km}$, between $\mathrm{H}-13$ and H-07 (Fig. 12c). The secondary maximum further 
(a) $\mathrm{mAF}$

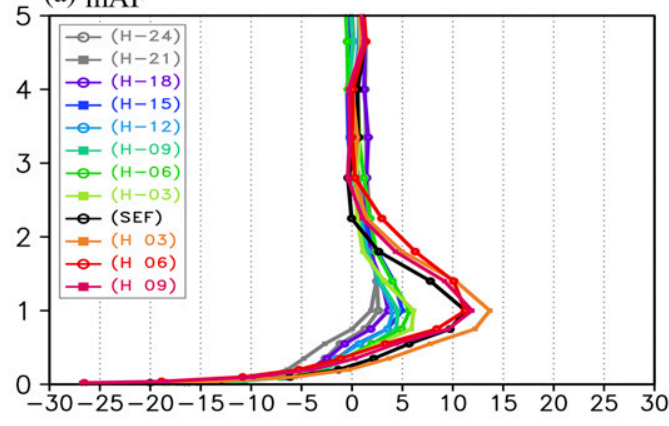

(c) $\overline{A F}=\mathrm{mAF}+\mathrm{eAF}$

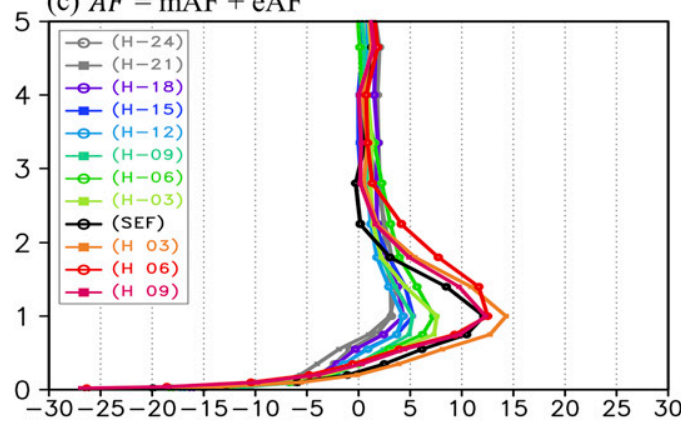

(e) $\overline{F_{r}}+\mathrm{mAF}$

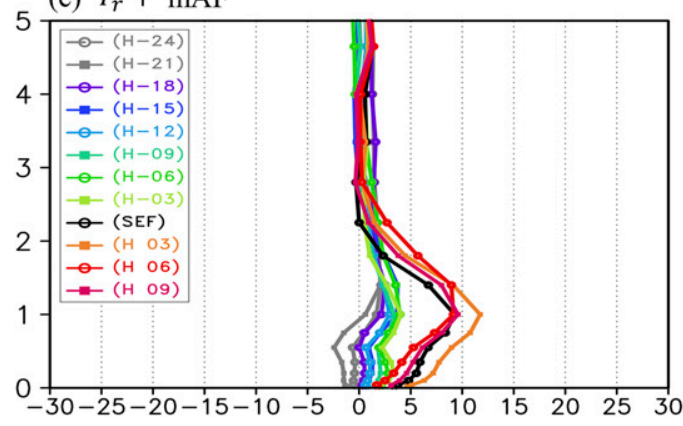

(b) eAF

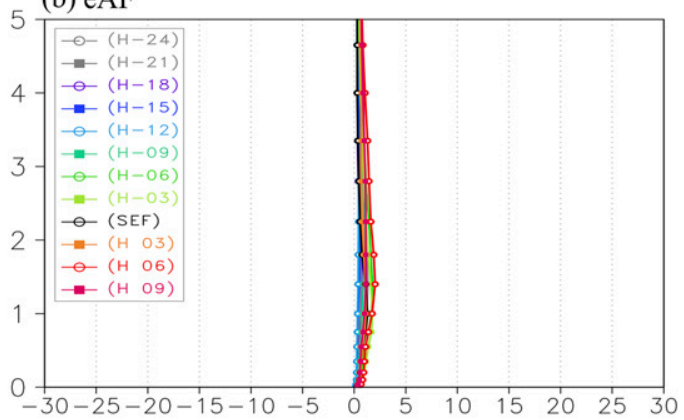

(d) $\overline{F_{r}}$

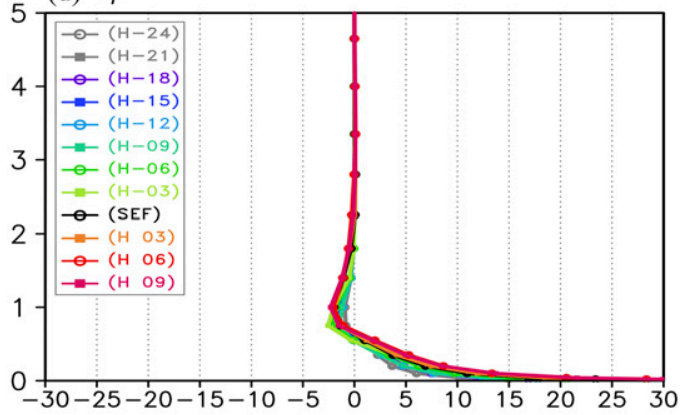

(f) $\bar{F}_{r}+\mathrm{mAF}+\mathrm{eAF}$

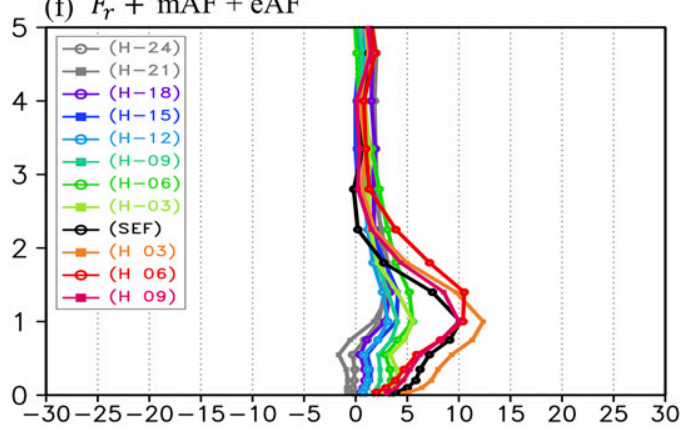

FIG. 11. As in Fig. 10, except abscissa now denotes the forcing terms of the material derivative of the radial-mean radial velocity prior to and just after SEF [Eqs. (2) and (3); $\mathrm{m} \mathrm{s}^{-1} \mathrm{~h}^{-1}$ ]. The (a) mAF [Eq. (4)]; (b) eAF [Eq. (5)]; (c) $\langle\mathrm{AF}\rangle$, the sum of mAF and eAF; (d) effects due to the PBL and surface parameterizations $\overline{F_{r}}$; (e) $\overline{F_{r}}+$ mAF; and (f) $\overline{F_{r}}+\mathrm{mAF}+\mathrm{eAF}$ [the sum of all the forcing terms in Eq. (2)].

strengthens and radially broadens in the boundary inflow layer when it approaches the SEF time $(\mathrm{H}-07-\mathrm{H}-01$; Fig. 12e). Within this inflow layer, the competing effect between these two terms explains how tangential winds swell up in the SEF region. Above the boundary inflow layer, the positive contribution from the total vertical advection concentrates in the SEF region and extends radially inward to about $60 \mathrm{~km}$ (Figs. 12b,d,f). The radially interior portion of the positive vertical advective effect is countered by the strengthening negative impact associated with the outflow just atop the boundary inflow layer. The positive vertical advection, which maximizes just above the inflow layer, acts to increase the tangential winds above the boundary inflow layer in the SEF region.
As shown in our two previous works, the broadening tangential wind outside the eyewall and the tangential wind jet of the outer eyewall are particularly evident in the lowest $2-3 \mathrm{~km}$. Interestingly, the tangential wind budget analysis shows two distinct processes to increase the tangential wind in the SEF region: namely, the positive competing effect between $-\bar{u} \overline{\zeta_{a}}$ and $\bar{F}_{\lambda}$ in the boundary inflow layer and the positive total vertical advective effect above the inflow layer. Figure 13 shows the radial distribution of each term on the righthand side of Eq. (1), and the depth averages below $1 \mathrm{~km}$ (the boundary inflow layer) and between 1 and $2.5 \mathrm{~km}$ are presented in the left and right panels, respectively. For convenience, the minus sign of the PBL 

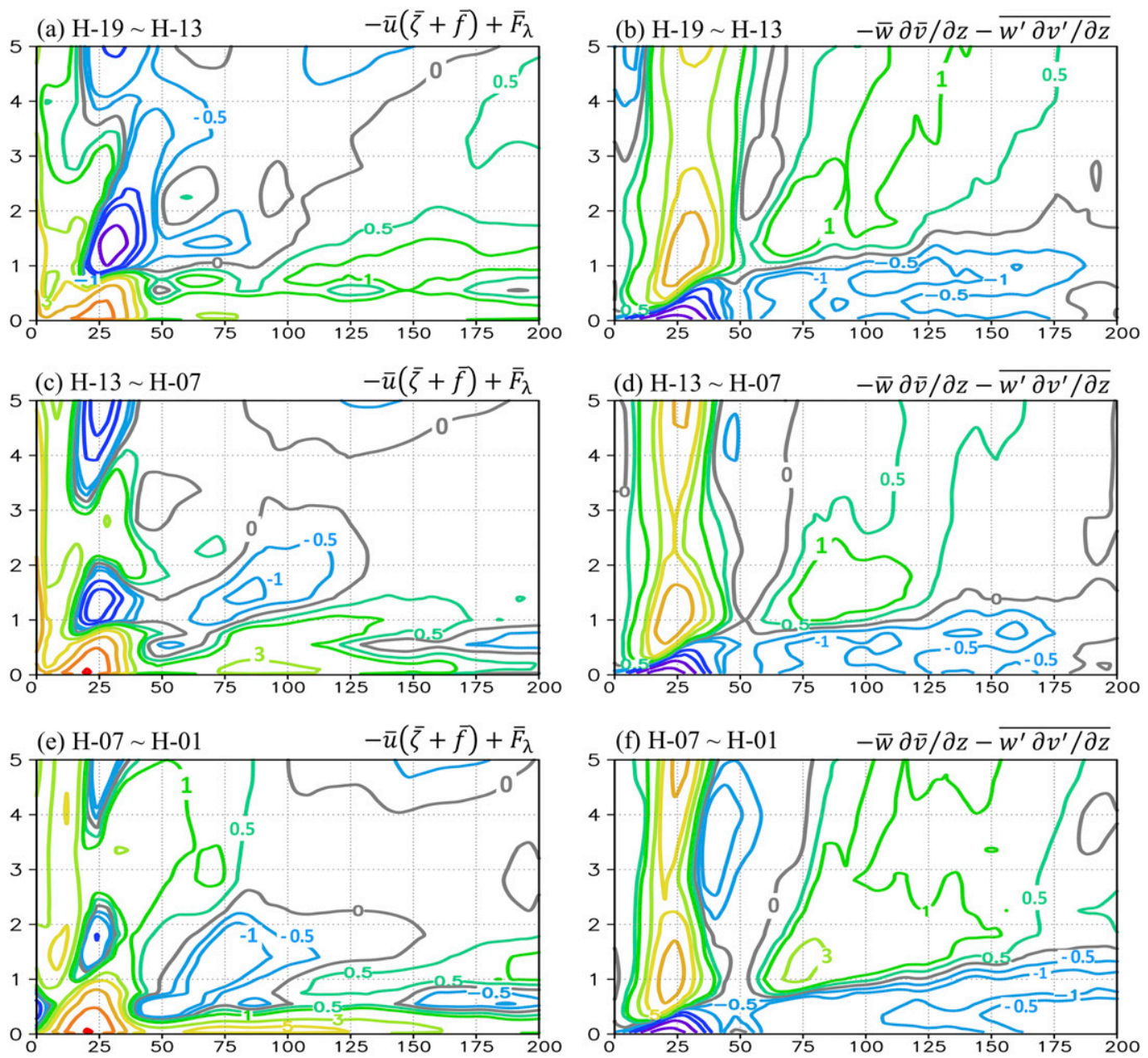

Contours: $-60,-40,-20,-10,-5,-3,-1,-0.5,0,0.5,1,3,5,10,20,40,60,80,100\left(m^{-1} h^{-1}\right)$

FIG. 12. As in Fig. 7, but showing the azimuthal-mean tangential wind tendency from the (a),(c),(e) sum of mean radial flux of absolute vorticity and PBL effect and (b),(d),(f) total vertical advection.

parameterization tendency $-\bar{F}_{\lambda}$ is plotted. In the SEF region, Fig. 13 clearly demonstrates the operation of these two distinct processes for increasing the mean tangential wind within and above the boundary layer inflow layer.

Within the boundary layer inflow layer, the small contributions of the total vertical advection and the eddy radial vorticity flux nearly cancel one another. The result of the two leading terms, as represented by the difference between the blue and the aqua curves, broadly reflects the simulated evolution of tangential wind outside the eyewall: 1) the broadening of the swirling circulation outside the eyewall between H-19 and H-13 (cf. Figs. 6a and 13a) and, later on, 2) the elevated winds concentrating in the SEF region (Figs. 6b,c and $13 \mathrm{c}, \mathrm{e})$. While the $\bar{F}_{\lambda}$ term retains its radial shape, an increase of $-\bar{u} \overline{\zeta_{a}}$ emerges between 75 and $150 \mathrm{~km}$
(Figs. 13a,c,e), thereby enhancing the secondary peak of their competing effect as shown in Figs. 12c and 12e.

3) THE ROLE OF THE BROADENING TANGENTIAL WIND FIELD IN THE RADIAL DISTRIBUTION OF THE MEAN RADIAL FLUX OF ABSOLUTE VORTICITY IN THE BOUNDARY INFLOW LAYER

To understand how $-\bar{u} \overline{\zeta_{a}}$ produces stronger tendency in the SEF region, the generalized Coriolis term $(-\bar{u} v / r$; associated equivalently with the curvature vorticity term) is shown in Figs. 13a, 13c, and 13e. Because of the limited contribution of planetary vorticity $f$ to absolute vorticity, one can infer the mean radial flux of shear vorticity $(-\bar{u} \partial \bar{v} / \partial r$; the shear term) from the difference between $-\bar{u} \bar{v} / r$ (pink) and $-\bar{u} \overline{\zeta_{a}}$ (aqua). Outside the original eyewall, the radii where the positive $-\bar{u} \overline{\zeta_{a}}$ swells up are collocated with the locations 
(a) $\mathrm{H}-19 \sim \mathrm{H}-13$

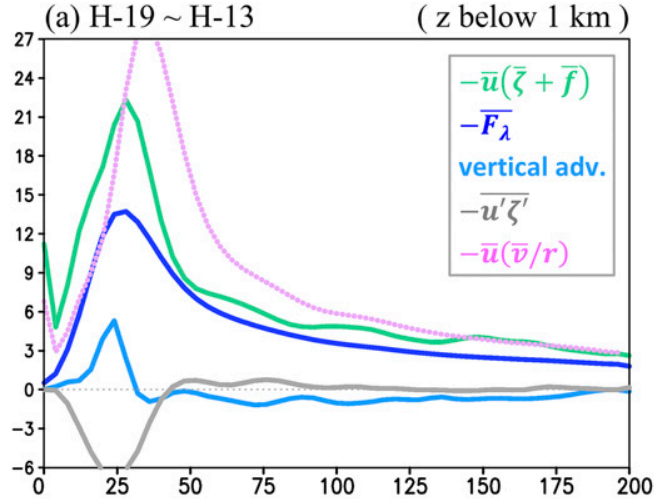

(c) $\mathrm{H}-13 \sim \mathrm{H}-07$

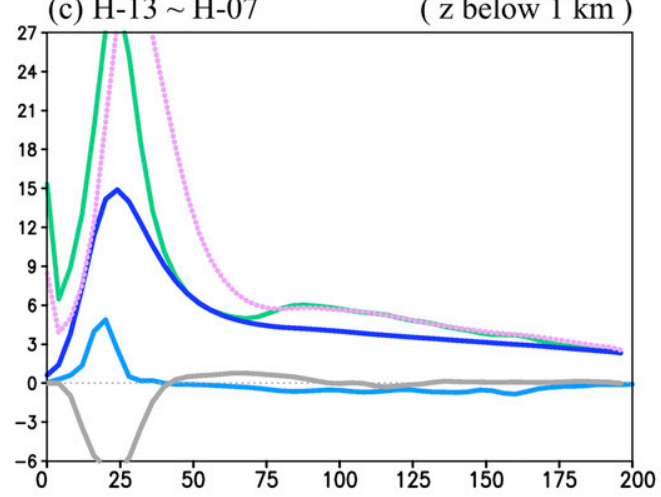

(e) $\mathrm{H}-07 \sim \mathrm{H}-01$

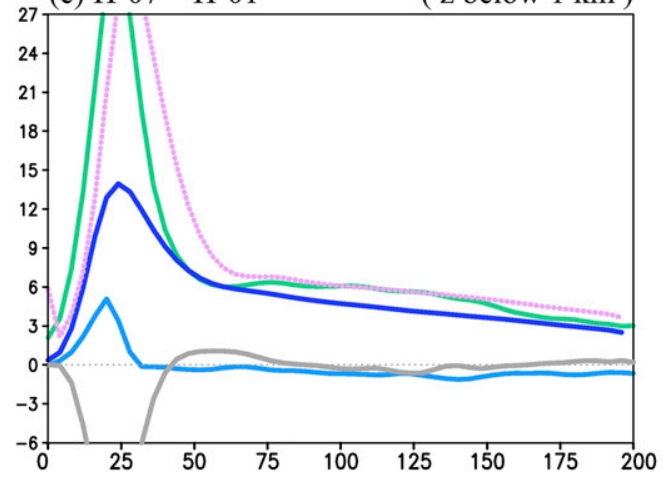

(b) $\mathrm{H}-19 \sim \mathrm{H}-13$

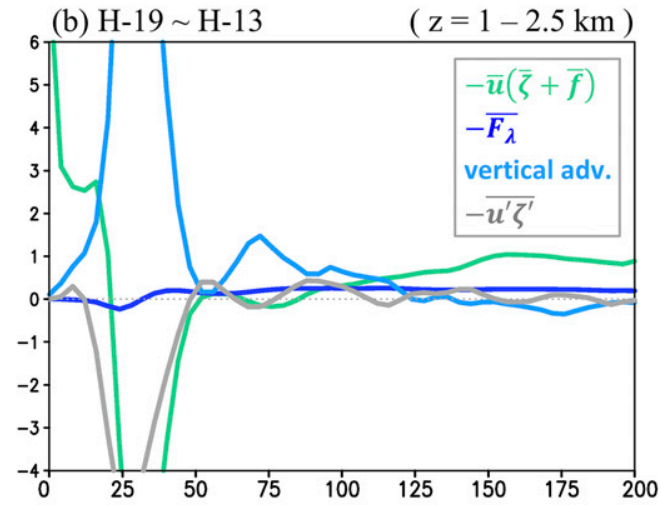

(d) $\mathrm{H}-13 \sim \mathrm{H}-07$

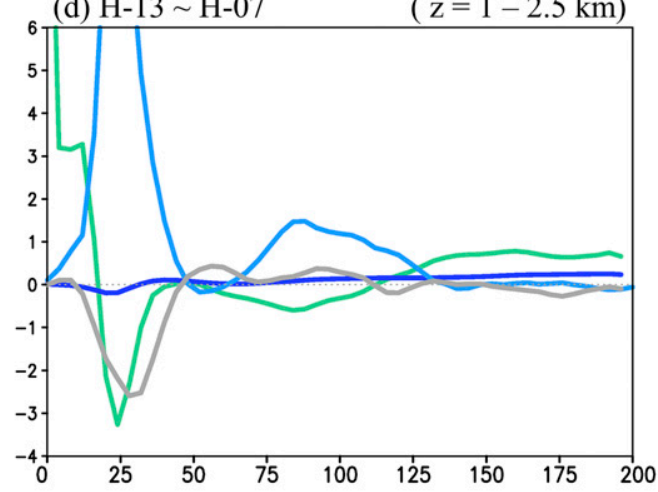

(f) $\mathrm{H}-07 \sim \mathrm{H}-01$

$(\mathrm{z}=1-2.5 \mathrm{~km})$

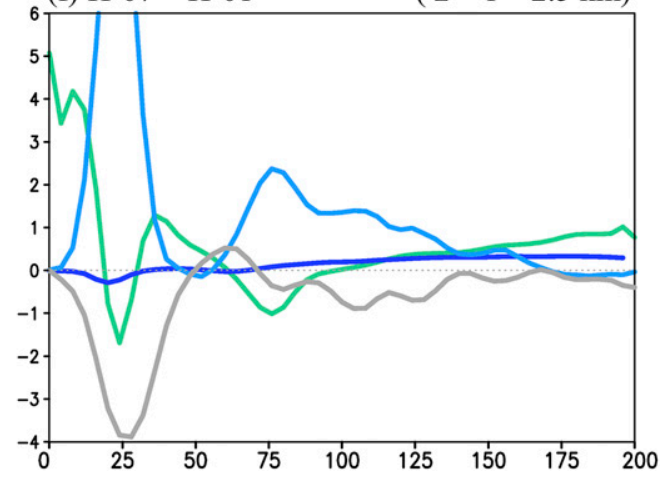

FIG. 13. The radial distribution of calculations on the azimuthal-mean tangential wind tendency $\left(\mathrm{m} \mathrm{s}^{-1} \mathrm{~h}^{-1}\right)$ for the depth averages (a),(c),(e) at the lowest $1 \mathrm{~km}$ (within the boundary inflow layer) and (b),(d),(f) between 1 and $2.5 \mathrm{~km}$. In the SEF region, the result demonstrates two distinct responsible processes for the increasing azimuthalmean tangential winds in the two vertical intervals: 1) the competing effect between the gain from the mean radial flux of absolute vorticity and the loss due to the PBL parameterization [(a), (c), and (e)], and 2) total vertical advection [(b), (d), and (f)]. The pink curve in (a), (c), and (e) additionally shows the mean radial flux of curvature vorticity. Different scales of the $y$ axis are applied to the two columns.

where the anticyclonic shear effect becomes subtle (Figs. 13c,e). The result demonstrates that while the curvature term dominates $-\bar{u} \overline{\zeta_{a}}$, the shear term further shapes the radial distribution of $-\bar{u} \overline{\zeta_{a}}$. The slightly greater positive curvature term on the smaller side of the broadening tangential wind field and the contrast between a negative effect of the shear term just outside the eyewall (around $40-75 \mathrm{~km}$ ) and the nearly zero influence of the shear term on the relatively flat wind field (outside $75 \mathrm{~km}$; cf. Figs. 3 and 13) together concentrate the positive $-\bar{u} \overline{\zeta_{a}}$ on the smallerradius side of the broadening tangential wind field, which is later on the location where the outer eyewall establishes. 


\section{$b$. The role of nonlinear unbalanced dynamics in the $S E F$}

The mean radial flux of absolute vorticity in the tangential wind equation is tied to the response to convective heating in the rainband(s) and the influence of surface friction. As mentioned in the introduction, the balanced secondary circulation in response to forcings of diabatic heating and momentum (such as surface friction) can be estimated by the Sawyer-Eliassen equations, providing a zeroth-order explanation for the evolving gradient winds. However, considering the increasing agradient component in the secondary azimuthalmean tangential wind jet, together with the fact that the elevated winds in the SEF region are found to be mainly composed of supergradient winds (Figs. 2-4), it stands to reason that dynamical processes in addition to the axisymmetric balance response to the heating and momentum forcings (i.e., unbalanced dynamics referred by this and other earlier studies) should play an important role in SEF. This viewpoint is supported by the findings of Slocum et al. (2014) and Abarca and Montgomery (2014), the latter of whom demonstrated that in the boundary layer the mean radial flux of absolute vorticity due to inflow obtained from the Sawyer-Eliassen equations does not fully compensate the loss caused by surface friction.

The unbalanced dynamics in relation to the frictional boundary layer processes has been articulated in previous studies (e.g., Smith et al. 2009; Bui et al. 2009; HMW12; Abarca and Montgomery 2013; Slocum et al. 2014; Smith and Montgomery 2016). In the tangential momentum equation, the frictional drag acts to decelerate the azimuthal-mean tangential wind [Eq. (1); $\bar{F}_{\lambda}$ in Figs. 7a,c,e and 10a], producing negative (radially inward) $\mathrm{mAF}$ in the material derivative of the azimuthal-mean radial velocity [Eqs. (2)-(4)] near the surface in the outer core of the vortex (e.g., Figs. 11a,c). The negative (subgradient) $\mathrm{mAF}$ acts to accelerate air parcels toward the circulation center and consequently to generate strong inflow. The inflow enhanced by the unbalanced dynamics, on the other hand, accelerates tangential winds via the mean radial flux of absolute vorticity (Figs. 7b,d,f and 10b), creating a flattened outer tangential wind profile. As discussed in section $6 \mathrm{a}(3)$, this flattened outer tangential wind along with the strong boundary layer inflow concentrates the increase of the azimuthal-mean tangential wind around the SEF region. The positive maximum of $-\bar{u} \overline{\zeta_{a}}$ around the SEF region strengthens in the boundary inflow layer of the SEF region as the SEF time approaches and maximizes near the surface. The latter infers the dominant role of unbalanced dynamics in producing the kinematic structure toward the SEF.
The unbalanced dynamics can also have an impact on increasing tangential winds above the boundary inflow layer via the vertical advection of enhanced momentum generated within the boundary layer. In the SEF region, the azimuthal-mean centrifugal and Coriolis forces associated with the increasing swirling wind start to considerably outweigh the mean pressure gradient force at the lowest $2 \mathrm{~km}$, except for the near surface, about 1 day prior to SEF. This produces an increasing positive (radially outward) mAF (Figs. 2 and 12a; see also Fig. 7 of HMW12). As articulated in section 5b and HMW12, the increasing positive $\mathrm{mAF}$ is key to the concentration of the boundary layer convergence and therefore provides an effective means for moisture air parcels to erupt out of the boundary layer. The mean vertical advection, which contributes to the upward extension of the tangential wind jet, is the product of vertical velocity and a partial derivative of the tangential wind with respect to height (Figs. 10d and 8a,c,e; see also Montgomery and Smith 2017). The positive impact of mean vertical advection atop of the boundary layer is primarily confined vertically to the $1-2-\mathrm{km}$ level. The vertical advection effect is radially collocated with and vertically atop the maximum mAF (cf. Figs. 2 and 8a,c,e), showing a clear connection of the strong upward motion and the unbalanced dynamics. This low-level maximum of the vertical advection attaches to another maximum of mean vertical advection above $3 \mathrm{~km}$ (Fig. 8). The above results are a reminder of the close relationship between the upward motion in response to the condensational heating and that forced via the unbalanced dynamics within and just above the boundary layer.

Moreover, since the mean vertical advection of momentum is in a downgradient form, it has a tendency to smooth the vertical gradient of the azimuthal tangential wind. It can be logically deduced that this outcome hinders the upward transport of the tangential momentum and consequently suppresses the upward extension of the tangential jet in the SEF region. The diagnosed results show that the unbalanced dynamics plays an important role in the upward extension of the tangential jet. In the SEF region, the outflow enhanced by the positive $\mathrm{mAF}$ atop the boundary inflow layer can help maintain or increase the negative vertical gradient of the azimuthal-mean tangential wind via the mean radial flux of absolute vorticity (Figs. 7b,d,f). Furthermore, the vertical structure of tangential winds within and just above the TC boundary layer is influenced also by the PBL parameterization (Kepert 2012). We therefore conclude that PBL processes, and the interplay between the boundary layer and free atmosphere [as shown in Eqs. (1) and (2)], can affect the SEF: namely, the establishment of the tangential wind maximum in the 
boundary layer and the vertical extension of the tangential wind jet.

\section{Summary and future directions}

Based on the foregoing analysis of the azimuthalmean tangential wind tendency equation, the competing effects between the gain of the mean radial flux of absolute vorticity and the loss due to the PBL subgrid-scale processes within the boundary inflow layer of the SEF region have been quantified. The results show two distinct responsible processes for the increasing azimuthalmean tangential winds in the two vertical intervals: 1 ) the competing effect between the gain from the mean radial flux of absolute vorticity and the loss due to the PBL parameterization in the boundary inflow layer and 2) total vertical advection just above the boundary layer.

In the boundary layer, the evolving mean radial flux of absolute vorticity is the leading-order factor responsible for the increasing tangential wind in the SEF region. The diminishing anticyclonic shear term and the slightly greater curvature term on the smaller-radius side of the broadening tangential wind field cause the azimuthalmean tangential wind to swell up therein. The total vertical advective effect contributes to the vertical extension of the elevated swirling flow and the secondary tangential jet by bringing the greatest momentum within and near the top of the boundary layer farther upward. The eddy radial flux of absolute vorticity appears to inhibit the establishment of a tangential wind jet in the SEF region, as it makes a maximum positive contribution to the azimuthal-mean tangential wind tendency in the moat region, in opposition to the fact that tangential winds are reduced or preserved in the moat.

As to the diagnosed results of the material derivative of the azimuthal-mean radial wind, the present analysis reveals that, in the SEF region, the frictional drag varies little with time, but the positive agradient forces evaluated by the azimuthal-mean mass and wind fields start to progressively intensify around 1 day prior to SEF. This demonstrates that the increasing positive (radially outward) net radial force, which decelerates inflowing air parcels across the SEF region, is largely attributed to the development of the mean supergradient forces. The eddy agradient force (particularly the eddy centrifugal force) slightly reinforces the positive net radial force.

The budget analyses presented here provide new quantitative evidence in support of the unbalanced boundary layer pathway to SEF proposed by HMW12. In a spatially limited region outside of the primary eyewall, HMW12 proposed that there is a strengthening boundary layer inflow forced by a radially expanding tangential wind field of an aging vortex. On account of the emerging supergradient winds and a positive agradient force in the boundary layer, the boundary layer inflow is then rapidly decelerated across the SEF region. The arrest of inflowing air parcels generates a focused boundary layer convergence, forcing the boundary layer air to erupt up into the free atmosphere. These persistent dynamical processes provide a mechanical forcing mechanism that supports the development of deep convection outside the primary eyewall, eventually leading to SEF provided that the local conditions remain dynamically and thermodynamically favorable for the development of deep convection.

The role of unbalanced boundary layer dynamics in enhancing the tangential winds in the SEF region is a special focus of this study. We provide dynamical linkages to the elevated winds within and just above the boundary inflow layer. First, the near-surface subgradient forces in a broad radial range of the vortex's outer-core region can accelerate radial inflow and thus help broaden the outer swirling circulation, which then contributes to the elevated mean radial flux of absolute vorticity and consequently the elevated tangential winds in boundary inflow layer of the SEF region. Second, the upward motion enhanced by the developing supergradient force therein leads to increasing positive mean vertical of tangential winds $1-2 \mathrm{~km}$ above the boundary inflow layer in the SEF region.

Although understanding of the unbalanced and balanced processes in tropical cyclone vortices undergoing secondary eyewall formation has advanced from the axisymmetric perspective, the quantitative impacts of these aspects on SEF and their interaction with other processes, such as vertical shear and related kinematic and thermodynamic processes (e.g., Riemer et al. 2010; Tang and Emanuel 2010; Riemer and Montgomery 2011; Nolan and McGauley 2012) remain to be further investigated. In addition to the axisymmetric mean dynamics, the dynamical contribution from eddy processes (nonaxisymmetric waves and quasi-two-dimensional and Kolmogorov turbulence) to SEF is of interest also (e.g., Persing et al. 2013; Smith et al. 2017). Indeed, spiral rainbands and sporadic convective cells are usually quite active in the vortex's outer-core region prior to (or even well before) SEF. It has been suggested also that eddy activities, such as vortex Rossby waves (e.g., Montgomery and Kallenbach 1997; Qiu et al. 2010; Abarca and Corbosiero 2011), and dynamics associated with the external forcing at upper levels (e.g., Nong and Emanuel 2003; Leroux et al. 2013; Dai et al. 2017) may contribute to SEF.

From the perspective of observation data, the environmental conditions favoring SEF are relatively well understood (Kossin and Sitkowski 2009, 2012), while 
interactions between a TC vortex and the accompanied convective-scale features prior to SEF and during an eyewall replacement cycle remain unclear. Further observational analyses of the secondary eyewall formation phase, paying close attention to both boundary layer and internal vortex dynamics, are required for a more comprehensive understanding of the physical processes in real and simulated SEF events.

Acknowledgments. We thank the two anonymous reviewers for providing helpful and in-depth comments. The work of Y.-H. Huang and C.-C. Wu is supported by the Ministry of Science and Technology of Taiwan through Grants MOST 105-2628-M-002-001 and 1062111-M-002-013-MY3. MTM acknowledges the support of NSF AGS-1313948, Office of Naval Research N0001417WX00336, and the U.S. Naval Postgraduate School. The views expressed herein are our own and do not reflect sponsoring agencies or institutions.

\section{REFERENCES}

Abarca, S. F., and K. L. Corbosiero, 2011: Secondary eyewall formation in WRF simulations of Hurricanes Rita and Katrina (2005). Geophys. Res. Lett., 38, L07802, https://doi.org/ 10.1029/2011GL047015.

_ , and M. T. Montgomery, 2013: Essential dynamics of secondary eyewall formation. J. Atmos. Sci., 70, 3216-3230, https://doi.org/10.1175/JAS-D-12-0318.1.

—_ and - 2014: Departures from the axisymmetric balance dynamics during secondary eyewall formation. J. Atmos. Sci., 71, 3723-3738, https://doi.org/10.1175/JAS-D-14-0018.1.

—, and - 2015: Are eyewall replacement cycles governed largely by axisymmetric balance dynamics? J. Atmos. Sci., 72, 82-87, https://doi.org/10.1175/JAS-D-14-0151.1.

- - - , and J. C. McWilliams, 2015: The azimuthally averaged boundary layer structure of a numerically simulated major hurricane. J. Adv. Model. Earth Syst., 7, 1207-1219, https://doi.org/ 10.1002/2015MS000457.

,-- , S. A. Braun, and J. Dunion, 2016: On the secondary eyewall formation of Hurricane Edouard (2014). Mon. Wea. Rev., 144, 3321-3331, https://doi.org/10.1175/MWR-D-15-0421.1.

Bell, M. M., M. T. Montgomery, and W.-C. Lee, 2012: An axisymmetric view of eyewall evolution in Hurricane Rita (2005). J. Atmos. Sci., 69, 2414-2432, https://doi.org/10.1175/ JAS-D-11-0167.1.

Black, M. L., and H. E. Willoughby, 1992: The concentric eyewall cycle of Hurricane Gilbert. Mon. Wea. Rev., 120, $947-$ 957, https://doi.org/10.1175/1520-0493(1992)120<0947: $\mathrm{TCECOH}>2.0 . \mathrm{CO} ; 2$.

Braun, S. A., P. A. Newman, and G. M. Heymsfield, 2016: NASA's Hurricane and Severe Storm Sentinel (HS3) investigation. Bull. Amer. Meteor. Soc., 97, 2085-2102, https://doi.org/10.1175/ BAMS-D-15-00186.1.

Bui, H. H., R. K. Smith, and M. T. Montgomery, 2009: Balanced and unbalanced aspects of tropical cyclone intensification. Quart. J. Roy. Meteor. Soc., 135, 1715-1731, https://doi.org/10.1002/qj.502.

Corbosiero, K. L., and J. Molinari, 2002: The effects of vertical wind shear on the distribution of convection in tropical cyclones. Mon. Wea. Rev., 130, 2110-2123, https://doi.org/ 10.1175/1520-0493(2002)130<2110:TEOVWS >2.0.CO;2.

Dai, Y., S. J. Majumdar, and D. S. Nolan, 2017: Secondary eyewall formation in tropical cyclones by outflow-jet interaction. J. Atmos. Sci., 74, 1941-1958, https://doi.org/10.1175/ JAS-D-16-0322.1.

Didlake, A. C., and R. A. Houze Jr., 2011: Kinematics of the secondary eyewall observed in Hurricane Rita (2005). J. Atmos. Sci., 68, 1620-1636, https://doi.org/10.1175/2011JAS3715.1.

, G. H. Heymsfield, P. D. Reasor, and S. R. Guimond, 2017: Concentric eyewall asymmetries in Gonzalo (2014) observed by airborne radar. Mon. Wea. Rev., 145, 729-749, https:// doi.org/10.1175/MWR-D-16-0175.1.

Eliassen, A., 1951: Slow thermally or frictionally controlled meridional circulation in a circular vortex. Astrophys. Norv., 5, 19-60.

Elsberry, R. L., and P. A. Harr, 2008: Tropical Cyclone Structure (TCS08) field experiment: Science basis, observational platforms, and strategy. Asia-Pac. J. Atmos. Sci., 44, 209-231.

Fang, J., and F. Zhang, 2012: Effect of beta shear on simulated tropical cyclones. Mon. Wea. Rev., 140, 3327-3346, https:// doi.org/10.1175/MWR-D-10-05021.1.

Hack, J. J., and W. H. Schubert, 1986: Nonlinear response of atmospheric vortices to heating by organized cumulus convection. J. Atmos. Sci., 43, 1559-1573, https://doi.org/10.1175/ 1520-0469(1986)043<1559:NROAVT>2.0.CO;2.

Hawkins, J. D., and M. Helveston, 2008: Tropical cyclone multiple eyewall characteristics. 28th Conf. on Hurricanes and Tropical Meteorology, Orlando, FL, Amer. Meteor. Soc., P1.7, https:// ams.confex.com/ams/26HURR/techprogram/paper_76084.htm.

Hence, D. A., and R. A. Houze Jr., 2012: Vertical structure of tropical cyclones with concentric eyewalls as seen by the TRMM Precipitation Radar. J. Atmos. Sci., 69, 1021-1036, https://doi.org/10.1175/JAS-D-11-0119.1.

Hodyss, D., and D. S. Nolan, 2007: Linear anelastic equations for atmospheric vortices. J. Atmos. Sci., 64, 2947-2959, https:// doi.org/10.1175/JAS3991.1.

Houze, R. A., Jr., 2010: Clouds in tropical cyclones. Mon. Wea. Rev., 138, 293-344, https://doi.org/10.1175/2009MWR2989.1.

, and Coauthors, 2006: The Hurricane Rainband and Intensity Change Experiment: Observations and modeling of Hurricanes Katrina, Ophelia, and Rita. Bull. Amer. Meteor. Soc., 87, 1503-1522, https://doi.org/10.1175/BAMS-87-11-1503.

- S. S. Chen, B. F. Smull, W.-C. Lee, and M. M. Bell, 2007: Hurricane intensity and eyewall replacement. Science, 315, 1235-1239, https://doi.org/10.1126/science.1135650.

Huang, Y.-H., M. T. Montgomery, and C.-C. Wu, 2012: Concentric eyewall formation in Typhoon Sinlaku (2008). Part II: Axisymmetric dynamical processes. J. Atmos. Sci., 69, 662-674, https://doi.org/10.1175/JAS-D-11-0114.1.

Judt, F., and S. S. Chen, 2010: Convectively generated potential vorticity in rainbands and formation of the secondary eyewall in Hurricane Rita of 2005. J. Atmos. Sci., 67, 3581-3599, https://doi.org/10.1175/2010JAS3471.1.

Kepert, J. D., 2012: Choosing a boundary layer parameterization for tropical cyclone modeling. Mon. Wea. Rev., 140, 14271445, https://doi.org/10.1175/MWR-D-11-00217.1.

2013: How does the boundary layer contribute to eyewall replacement cycles in axisymmetric tropical cyclones? J. Atmos. Sci., 70, 2808-2830, https://doi.org/10.1175/JAS-D-13-046.1. 2017: Time and space scales in the tropical cyclone boundary layer, and the location of the eyewall updraft. J. Atmos. Sci., 74, 3305-3323, https://doi.org/10.1175/JAS-D-17-0077.1. 
and D. S. Nolan, 2014: Reply to "Comments on 'How does the boundary layer contribute to eyewall replacement cycles in axisymmetric tropical cyclones?"' J. Atmos. Sci., 71, 46924704, https://doi.org/10.1175/JAS-D-14-0014.1.

Kossin, J. P., and M. Sitkowski, 2009: An objective model for identifying secondary eyewall formation in hurricanes. Mon. Wea. Rev., 137, 876-892, https://doi.org/10.1175/ 2008MWR2701.1.

- , and — 2012: Predicting hurricane intensity and structure changes associated with eyewall replacement cycles. Wea. Forecasting, 27, 484-488, https://doi.org/10.1175/WAF-D-11-00106.1.

Kuo, H.-C., W. H. Schubert, C.-L. Tsai, and Y.-F. Kuo, 2008: Vortex interactions and barotropic aspects of concentric eyewall formation. Mon. Wea. Rev., 136, 5183-5198, https:// doi.org/10.1175/2008MWR2378.1.

—_, C.-P. Chang, Y.-T. Yang, and H.-J. Jiang, 2009: Western North Pacific typhoons with concentric eyewalls. Mon. Wea. Rev., 137, 3758-3770, https://doi.org/10.1175/ 2009MWR2850.1.

Leroux, M.-D., M. Plu, D. Barbary, F. Roux, and P. Arbogast, 2013: Dynamical and physical processes leading to tropical cyclone intensification under upper-level trough forcing. J. Atmos. Sci., 70, 2547-2565, https://doi.org/10.1175/JAS-D-12-0293.1.

Menelaou, K., M. K. Yau, and Y. Martinez, 2014: Some aspects of the problem of secondary eyewall formation in idealized three-dimensional nonlinear simulations. J. Adv. Model. Earth Syst., 6, 491-512, https://doi.org/10.1002/2014MS000316.

Meng, Z., and F. Zhang, 2007: Test of an ensemble Kalman filter for mesoscale and regional-scale data assimilation. Part II: Imperfect model experiments. Mon. Wea. Rev., 135, 14031423, https://doi.org/10.1175/MWR3352.1.

$\longrightarrow$, and — 2008a: Test of an ensemble Kalman filter for mesoscale and regional-scale data assimilation. Part III: Comparison with 3DVar in a real-data case study. Mon. Wea. Rev., 136, 522-540, https://doi.org/10.1175/2007MWR2106.1.

—_, and —_, 2008b: Test of an ensemble Kalman filter for mesoscale and regional-scale data assimilation. Part IV: Comparison with 3DVar in a month-long experiment. Mon. Wea. Rev., 136, 3671-3682, https://doi.org/10.1175/ 2008MWR2270.1.

Montgomery, M. T., and R. J. Kallenbach, 1997: A theory for vortex Rossby waves and its application to spiral bands and intensity changes in hurricanes. Quart. J. Roy. Meteor. Soc., 123, 435-465, https://doi.org/10.1002/qj.49712353810.

, and R. K. Smith, 2017: On the applicability of linear axisymmetric dynamics in intensifying and mature tropical cyclones. Fluids, 2, 69, https://doi.org/10.3390/fluids2040069.

_ S. F. Abarca, R. K. Smith, C.-C. Wu, and Y.-H. Huang, 2014: Comments on "How does the boundary layer contribute to eyewall replacement cycles in axisymmetric tropical cyclones?" J. Atmos. Sci., 71, 4682-4691, https://doi.org/10.1175/ JAS-D-13-0286.1.

Nolan, D. S., and M. G. McGauley, 2012: Tropical cyclogenesis in wind shear: Climatological relationships and physical processes. Cyclones: Formation, Triggers and Control, K. Oouchi and H. Fudeyasu, Eds., Nova Science Publishers, 1-36.

- Y. Moon, and D. P. Stern, 2007: Tropical cyclone intensification from asymmetric convection: Energetics and efficiency. J. Atmos. Sci., 64, 3377-3405, https://doi.org/10.1175/ JAS3988.1.

Nong, S., and K. Emanuel, 2003: Concentric eyewalls in hurricanes. Quart. J. Roy. Meteor. Soc., 129, 3323-3338, https://doi.org/ 10.1256/qj.01.132.
Persing, J., M. T. Montgomery, J. McWilliams, and R. K. Smith, 2013: Asymmetric and axisymmetric dynamics of tropical cyclones. Atmos. Chem. Phys., 13, 12 299-12 341, https://doi.org/ 10.5194/acp-13-12299-2013.

Qiu, X., and Z.-M. Tan, 2013: The roles of asymmetric inflow forcing induced by outer rainbands in tropical cyclone secondary eyewall formation. J. Atmos. Sci., 70, 953-974, https:// doi.org/10.1175/JAS-D-12-084.1.

,$- \ldots$, and Q. Xiao, 2010: The roles of vortex Rossby waves in hurricane secondary eyewall formation. Mon. Wea. Rev., 138, 2092-2109, https://doi.org/10.1175/2010MWR3161.1.

Riemer, M., and M. T. Montgomery, 2011: Simple kinematic models for the environmental interaction of tropical cyclones in vertical wind shear. Atmos. Chem. Phys., 11, 9395-9414, https://doi.org/10.5194/acp-11-9395-2011.

$\_,-$_ and M. A. Nicholls, 2010: A new paradigm for intensity modification of tropical cyclones: Thermodynamic impact of vertical wind shear on the inflow layer. Atmos. Chem. Phys., 10, 3163-3188, https://doi.org/10.5194/acp-10-3163-2010.

Rozoff, C. M., D. S. Nolan, J. P. Kossin, F. Zhang, and J. Fang, 2012: The roles of an expanding wind field and inertial stability in tropical cyclone secondary eyewall formation. J. Atmos. Sci., 69, 2621-2643, https://doi.org/10.1175/JAS-D-11-0326.1.

Schubert, W. H., and J. J. Hack, 1982: Inertial stability and tropical cyclone development. J. Atmos. Sci., 39, 1687-1697, https://doi.org/10.1175/1520-0469(1982)039<1687:ISATCD > 2.0. $\mathrm{CO} ; 2$.

Shapiro, L. J., and H. E. Willoughby, 1982: The response of balanced hurricanes to local sources of heat and momentum. J. Atmos. Sci., 39, 378-394, https://doi.org/10.1175/15200469(1982)039<0378:TROBHT>2.0.CO;2.

Sitkowski, M., J. P. Kossin, and C. M. Rozoff, 2011: Intensity and structure changes during hurricane eyewall replacement cycles. Mon. Wea. Rev., 139, 3829-3847, https://doi.org/10.1175/ MWR-D-11-00034.1.

,,--- , and J. A. Knaff, 2012: Hurricane eyewall replacement cycle thermodynamics and the relict inner eyewall circulation. Mon. Wea. Rev., 140, 4035-4045, https://doi.org/ 10.1175/MWR-D-11-00349.1.

Skamarock, W. C., J. B. Klemp, J. Dudhia, D. O. Gill, D. M. Barker, W. Wang, and J. G. Powers, 2005: A description of the Advanced Research WRF version 2. NCAR Tech. Note NCAR/ TN-4681ST, 88 pp., https://doi.org/10.5065/D6DZ069T.

Slocum, C. J., G. J. Williams, R. K. Taft, and W. H. Schubert, 2014: Tropical cyclone boundary layer shocks. arXiv, http://arxiv. org/abs/1405.7939.

Smith, R. K., and M. T. Montgomery, 2015: Toward clarity on tropical cyclone intensification. J. Atmos. Sci., 72, 3020-3031, https://doi.org/10.1175/JAS-D-15-0017.1.

— and - 2016: The efficiency of diabatic heating and tropical cyclone intensification. Quart. J. Roy. Meteor. Soc., 142, 20812086, https://doi.org/10.1002/qj.2804.

_,- , and S. V. Nguyen, 2009: Tropical cyclone spin-up revisited. Quart. J. Roy. Meteor. Soc., 135, 1321-1335, https:// doi.org/10.1002/qj.428.

__, J. A. Zhang, and M. T. Montgomery, 2017: The dynamics of intensification in a Hurricane Weather Research and Forecasting simulation of Hurricane Earl (2010). Quart. J. Roy. Meteor. Soc., 143, 293-308, https://doi.org/10.1002/qj.2922.

Sun, Y. Q., Y. Jiang, B. Tan, and F. Zhang, 2013: The governing dynamics of the secondary eyewall formation of Typhoon Sinlaku (2008). J. Atmos. Sci., 70, 3818-3837, https://doi.org/ 10.1175/JAS-D-13-044.1. 
Tang, B., and K. Emanuel, 2010: Midlevel ventilation's constraint on tropical cyclone intensity. J. Atmos. Sci., 67, 1817-1830, https://doi.org/10.1175/2010JAS3318.1.

Terwey, W. D., and M. T. Montgomery, 2008: Secondary eyewall formation in two idealized, full-physics modeled hurricanes. J. Geophys. Res., 113, D12112, https://doi.org/ 10.1029/2007JD008897.

Wang, H., C.-C. Wu, and Y. Wang, 2016: Secondary eyewall formation in an idealized tropical cyclone simulation: Balanced and unbalanced dynamics. J. Atmos. Sci., 73, 3911-3930, https://doi.org/10.1175/JAS-D-15-0146.1.

Wang, X., Y. Ma, and N. E. Davidson, 2013: Secondary eyewall formation and eyewall replacement cycles in a simulated hurricane: Effect of the net radial force in the hurricane boundary layer. J. Atmos. Sci., 70, 1317-1341, https://doi.org/ 10.1175/JAS-D-12-017.1.

Willoughby, H. E., and P. G. Black, 1996: Hurricane Andrew in Florida: Dynamics of a disaster. Bull. Amer. Meteor. Soc., 77, 543-549, https://doi.org/10.1175/1520-0477(1996)077<0543: $\mathrm{HAIFDO}>2.0 . \mathrm{CO} ; 2$

_ J. A. Clos, and M. G. Shoreibah, 1982: Concentric eyewalls, secondary wind maxima, and the evolution of the hurricane vortex. J. Atmos. Sci., 39, 395-411, https://doi.org/10.1175/ 1520-0469(1982)039<0395:CEWSWM > 2.0.CO;2.

— , F. D. Marks Jr., and R. J. Feinberg, 1984: Stationary and propagating convective bands in hurricanes. J. Atmos. Sci., 41,
3189-3211, https://doi.org/10.1175/1520-0469(1984)041<3189: SAMCBI $>2.0 . \mathrm{CO} ; 2$.

Wu, C.-C., G.-Y. Lien, J.-H. Chen, and F. Zhang, 2010: Assimilation of tropical cyclone track and structure based on the ensemble Kalman filter (EnKF). J. Atmos. Sci., 67, 3806-3822, https://doi.org/10.1175/2010JAS3444.1.

_, Y.-H. Huang, and G.-Y. Lien, 2012: Concentric eyewall formation in Typhoon Sinlaku (2008). Part I: Assimilation of T-PARC data based on the ensemble Kalman filter (EnKF). Mon. Wea. Rev., 140, 506-527, https://doi.org/ 10.1175/MWR-D-11-00057.1.

- - - , and Z. Tan, 2015: Secondary eyewall formation in tropical cyclones. Dynamics and Predictability of Large-Scale High-Impact Weather and Climate Events, J. Li et al., Eds., Cambridge University Press, 168-175.

Yang, Y.-T., H.-C. Kuo, E. A. Hendricks, and M. S. Peng, 2013: Structural and intensity changes of concentric eyewall typhoons in the western North Pacific basin. Mon. Wea. Rev., 141, 2632-2648, https://doi.org/10.1175/MWR-D-12-00251.1.

Zhou, X., and B. Wang, 2013: Large-scale influences on secondary eyewall size. J. Geophys. Res. Atmos., 118, 11 088-11 097, https://doi.org/10.1002/jgrd.50605.

Zhu, Z., and P. Zhu, 2014: The role of outer rainband convection in governing the eyewall replacement cycle in numerical simulations of tropical cyclones. J. Geophys. Res. Atmos., 119, 8049-8072, https://doi.org/10.1002/2014JD021899. 